\title{
Biopharmaceutical Modeling of Drug Supersaturation During Lipid-Based Formulation Digestion Considering an Absorption Sink
}

\author{
Cordula Stillhart • Georgios Imanidis • Brendan T. Griffin • Martin Kuentz
}

Received: 29 January 2014 / Accepted: 3 June 2014 / Published online: 25 June 2014

(C) Springer Science+Business Media New York 2014

\begin{abstract}
Purpose In vitro lipolysis is widely utilized for predicting in vivo performance of oral lipid-based formulations (LBFs). However, evaluation of LBFs in the absence of an absorption sink may have limited in vivo relevance. This study aimed at employing biopharmaceutical modeling to simulate LBF digestion and drug supersaturation in a continuous absorptive environment.

Methods Three fenofibrate-loaded LBFs were characterized in vitro (dispersion and lipolysis) and drug precipitation was monitored using in-line Raman spectroscopy. In vitro data were combined with pharmacokinetic data derived from an in vivo study in pigs to simulate intestinal LBF transit. This biopharmaceutical model allowed calculation of lipolysis-triggered drug supersaturation while drug and lipolysis products are absorbed from the intestine.

Results The biopharmaceutical model predicted that, in a continuous absorption environment, fenofibrate supersaturation was considerably lower compared to in vitro lipolysis (non-sink). Hence, the extensive drug precipitation observed in vitro was predicted to be unlikely in vivo. The absorption of lipolysis products increased drug supersaturation, but drug precipitation was unlikely for highly permeable drugs.
\end{abstract}

Conclusions Biopharmaceutical modeling is a valuable approach for predicting LBFs performance in vivo. In the absence of in vitro tools simulating absorptive conditions, modeling strategies should be further considered.

C. Stillhart · G. Imanidis

Department of Pharmaceutical Sciences, University of Basel

Klingelbergstrasse 50, 4056 Basel, Switzerland

C. Stillhart • G. Imanidis $\cdot$ M. Kuentz $(\bowtie)$ Institute of Pharma Technology, School of Life Sciences, University of Applied Sciences and Arts Northwestern Switzerland

Gründenstrasse 40, 4I32 Muttenz, Switzerland

e-mail: Martin.Kuentz@fhnw.ch

B. T. Griffin

School of Pharmacy, University College Cork, Cork, Ireland
KEYWORDS Lipid-based formulation · Lipolysis · Absorption sink · Supersaturation · Biopharmaceutical modeling

\section{INTRODUCTION}

Low aqueous solubility and poor dissolution in the gastrointestinal (GI) fluids are major reasons for limited oral absorption of poorly water-soluble compounds. Such drug candidates may therefore exhibit low and variable oral bioavailability, which hinders effective drug development. Lipid-based formulations $(\mathrm{LBFs})$ are gaining increasing interest as a drug delivery strategy for poorly water-soluble compounds $(1,2)$. The improvement in oral bioavailability is obtained via several mechanisms. The critical dissolution step is circumvented and the apparent drug solubility in GI fluids is generally increased in presence of lipidic excipients. Additionally, lipid excipients may increase drug permeability across the intestinal membrane and promote drug uptake via the intestinal lymphatics (1).

The improvement of oral absorption from LBFs is also significantly influenced by the fate of the LBF in the GI tract. Digestion of lipidic excipients by endogenous lipases is particularly critical due to the formation of more polar lipolysis products (i.e., monoglycerides (MGs) and fatty acids (FAs)) and the potential of drug precipitation. Such drug precipitation in vivo is undesirable, as subsequent re-dissolution is likely to delay and/or reduce the overall extent of drug absorption. The solid-state properties of the precipitate that forms in vivo therefore become critical. While it has been shown for some drugs that the formation of an amorphous precipitate may be favorable in terms of higher apparent solubility and facilitated re-dissolution, this is not the case in all drug types $(4,5)$. There is also growing evidence that the kinetics of precipitation is a significant determinant of LBF performance in viwo (6). From a biopharmaceutical perspective, the initial induction period of drug nucleation is highly relevant, as during this stage the actual drug concentration exceeds equilibrium solubility, i.e., 
the drug is supersaturated. Although being thermodynamically unstable, this supersaturated state promotes absorptive flux across the intestinal membrane, and hence, the longer the induction period, the more drug can be absorbed (7). To maximize the biopharmaceutical benefit, supersaturation should be high enough to enhance drug uptake, but without inducing nucleation of drug particles in a physiologically relevant time span.

An in vitro lipolysis assay is commonly employed to study the impact of digestion on the drug solubilization capacity of LBFs $(8,9)$. Formulation digestion is performed using simulated intestinal medium and pancreatic enzymes, and the digestion is monitored by direct titration of the liberated FAs. Several reports categorized LBFs in terms of their propensity for lipolysis-triggered precipitation in vitro, but the in vivo performance was successfully predicted only in few studies and mainly rank-order correlations have been observed (10). Strategies are therefore necessary to improve accuracy of in vitro - in vivo correlation (IVIVG) for LBFs. It is hypothesized that a major limitation of the current in vitro lipolysis test is the absence of an absorptive sink compartment. Given that the current test is a closed system (i.e., no absorption sink), the drug concentrations may differ from those observed in vivo, especially for highly permeable compounds, which are continuously removed via absorption.

In an attempt to improve the relevance to the in vivo situation, Shi et al. developed a biphasic system, comprising an aqueous buffer and an organic solvent as acceptor phase (11). Although a good IVIVC was obtained, this approach was only suitable for simulating LBF dispersion in the absence of biorelevant media. Alternative approaches using artificial or biological membranes (12) have also been applied, but in addition to the increased model complexity, critical parameters, such as the appropriate filter membrane selection (regarding molecular cut-off) as well as the small surface area for absorption require careful consideration.

Computational methods are a promising alternative for simulating complex biopharmaceutical processes. Most recently, Fei et al. presented such an in silico approach for modeling the GI performance of self-emulsifying formulations (13). Thus, in vitro dissolution data (without digestive enzymes) were incorporated in a biopharmaceutical model to simulate simultaneous formulation dispersion and drug absorption. The resulting in silico model suitably predicted oral bioavailability in humans. The LBFs comprised mostly surfactants (SFs) with comparatively low amounts of digestible lipids, and hence, LBF dispersion was considered the most critical step with respect to drug precipitation. However, in presence of larger amounts of digestible lipids, formulation digestion may become increasingly critical. For such formulations, the possibility to model formulation lipolysis and lipolysistriggered drug precipitation is highly desirable. Recently we introduced a mathematical nucleation and growth model that described drug precipitation during formulation lipolysis (14). This model was based on in vitro lipolysis data that were acquired under standard conditions, i.e., without an absorption sink.

The purpose of the present study was to develop a computational approach to predict the impact of formulation digestion in an absorption environment. To this end, using both in vitro and in vivo data, we developed an in silico biopharmaceutical model of LBF digestion and simultaneous drug absorption. Intestinal drug concentration profiles were derived from an in vivo study (in pigs) following oral administration of three fenofibrate-loaded LBFs (15). A mathematical model simulating the time evolution of drug solubility in the intestine was developed based on in vitro solubility and in vitro lipolysis data. The biopharmaceutical model was then used to calculate intralumenal drug supersaturation to predict the likelihood of lipolysis-triggered drug precipitation in vivo.

\section{MATERIALS AND METHODS}

\section{Materials}

Fenofibrate (2-[4-(4-chlorobenzoyl) phenoxy]-2-methylpropionic acid 1-methylethyl ester, $>99 \%$ ), olive oil Ph. Eur., Tween ${ }^{\circledR} 85$, Trizma ${ }^{\circledR}$ maleate, calcium chloride dihydrate $(>99 \%)$, pancreatin (from porcine pancreas, 8xUSP specifications), chloroform, and acetonitrile were obtained from Sigma-Aldrich Chemie GmbH (Buchs, Switzerland), and sodium chloride (>99\%) from Carl Roth GmbH (Karlsruhe, Germany). Lipoid ${ }^{\circledR}$ E PC S (phosphatidylcholine from egg yolk) was supplied by Lipoid GmbH (Ludwigshafen, Germany), sodium taurodeoxycholate by Prodotti Chimici e Alimentari S.p.A. (Basaluzzo, Italy), sodium hydroxide $1 \mathrm{M}$ by Scharlab S.L. (Sentmenat, Spain), and 4bromophenylboronic acid $\left(>95.0^{\circ}\right.$ ) by AK Scientific (Union City, CA, USA). Miglyol ${ }^{\circledR} 812 \mathrm{~N}$ was purchased from Hänseler AG (Herisau, Switzerland), and Cremophor ${ }^{\circledR}$ RH 40 from BASF AG (Ludwigshafen, Germany). Purified water was prepared using an Arium ${ }^{\circledR} 61215$ water-purification system from Sartorius Stedim Biotech GmbH (Göttingen, Germany). Anotop $^{\circledR} 25$ Plus filters (aluminum oxide, $0.1 \mu \mathrm{m}$ ) were purchased from Whatman GmbH (Dassel, Germany) for nanofiltration.

Miglyol $812 \mathrm{~N}$ is a medium-chain triglyceride (TG) consisting of $57.9 \% \mathrm{w} / \mathrm{w}$ caprylic acid (C8), 41.2\% w/w capric acid (C10), 0.5\% w/w lauric acid (C12), and $0.1 \%$ $\mathrm{w} / \mathrm{w}$ caproic acid (C6) with an average molecular weight of $517 \mathrm{~g} / \mathrm{mol}$ (according to the certificate of analysis, Hänseler AG, Switzerland). Olive oil Ph. Eur. is a long-chain TG composed of oleic acid (C18, 80\%) and linoleic acid (C18, $\sim 20 \%$ ). Tween 85 is a polyoxyethylene sorbitan trioleate surfactant (HLB 11) and Cremophor RH 40 is polyoxyl 40 hydrogenated castor oil (HLB 15). 


\section{Preparation of Formulations}

The compositions of the three LBFs used in this study are summarized in Table I. The model LBFs comprised two SFs (Cremophor RH 40 and Tween 85) at a fixed ratio of 1:2 w/w. Formulation IIIB/IV was a surfactant-only system, whereas IIIA LC and IIIA MC additionally comprised an oil component of $40 \% \mathrm{w} / \mathrm{w}$ olive oil (long-chain TG) and $40 \%$ w/w Miglyol 812 (medium-chain TG), respectively.

The excipients were weighted and mixed until a clear solution was obtained. Fenofibrate was then incorporated into the formulations at $80 \mathrm{mg} / \mathrm{g}$, which corresponded to a saturation level of $56 \%, 83 \%$, and $77 \%$ in formulation IIIA LC, IIIA MC, and IIIB/IV, respectively (15). The blends were stirred at $50^{\circ} \mathrm{C}$ for $30 \mathrm{~min}$ and stored overnight at $37^{\circ} \mathrm{C}$ to ensure complete dissolution of the drug. All formulations were visually assessed for absence of undissolved drug particles prior to use.

\section{In Vitro Lipolysis}

The in vitro lipolysis test was performed as described in the literature (8). Briefly, $108 \mathrm{ml}$ of a micellar solution $(50 \mathrm{mM}$ Trizma maleate, $150 \mathrm{mM} \mathrm{NaCl}$, and $5 \mathrm{mM} \mathrm{CaCl}_{2} \cdot 2 \mathrm{H}_{2} \mathrm{O}$ (pH 7.5) containing $1.25 \mathrm{mM}$ phosphatidylcholine $(\mathrm{PC})$ and $5 \mathrm{mM}$ sodium taurodeoxycholate (NaTDG)) were heated in a thermostated glass vessel $\left(37.0 \pm 0.5^{\circ} \mathrm{C}\right)$ and the formulation $(3 \mathrm{~g})$ was added. The mixture was stirred (450 rpm, $10 \mathrm{~min}$ ) for complete dispersion, thermal equilibration, and $\mathrm{pH}$ adjustment to 7.5. Digestion was initiated with the addition of $12 \mathrm{ml}$ pancreatin extract (final nominal lipase activity: 1000 tributyrin units per $\mathrm{ml}$ ) obtained from porcine pancreatin powder (8).

The free FAs produced during lipolysis were titrated using $1 \mathrm{M} \mathrm{NaOH}$ to maintain $\mathrm{pH} 7.5$ using a $\mathrm{pH}$-stat apparatus (842 Titrando and 800 Dosino, Metrohm AG, Switzerland) with the Tiamo ${ }^{\circledR} 1.2$ software package (Metrohm AG, Switzerland). Lipolysis was allowed to proceed for $60 \mathrm{~min}$. To determine the $\mathrm{NaOH}$ consumption caused by digestion of the blank digestion medium, lipolysis experiments were run with pure digestion medium without formulation. In vitro lipolysis was assayed in triplicate with each formulation.

To determine the total amount of FAs released during formulation digestion, a so-called "back-titration" was carried out as previously described (14). This was necessary because some FAs exist in their unionized state, according to the $\mathrm{pK}_{\mathrm{a}}$ value, leading to an underestimation of the total amount of FAs liberated.

The extent of formulation digestion was calculated according to:

Extent of digestion $=\frac{F A_{\text {titr }}^{\text {tot }}}{\text { Theor.amount of digestible FAs in } L B F}$ where $F A_{\text {titr }}^{\text {tot }}$ was the total amount of ionized and unionized FAs liberated by 60 min of digestion. The theoretical amount of digestible FAs in LBFs was calculated assuming that each TG and SF molecule liberated two FAs.

\section{Quantification of Drug Precipitation Using Raman Spectroscopy}

Raman spectroscopy was used to quantify fenofibrate precipitation during in vitro lipolysis as previously described (14). A multi-fiber Raman $\mathrm{P}^{h} \mathrm{AT}$ probe and a Raman RXN1 analyzer (Kaiser Optical Systems, Inc., Ann Arbor, MI, USA) equipped with a diode laser (wavelength of $785 \mathrm{~nm}$, laser power of $400 \mathrm{~mW}$ ) were used. A single spectrum was collected every $20 \mathrm{~s}$ (acquisition time $18.5 \mathrm{~s}$ ) with a resolution of $4 \mathrm{~cm}^{-1}$ using the $\mathrm{iC} \operatorname{Raman}^{\circledR}$ Instrument software (Version 3.0, Mettler-Toledo AutoChem Inc., Columbia, MD, USA).

To remove sources of non-linearity and spectral information that was uncorrelated with the concentration of the analyte, the spectra were subjected to the Savitzky-Golay polynomial derivative filter (14). The fingerprint spectral range of fenofibrate $\left(1000-1800 \mathrm{~cm}^{-1}\right)$ was used for drug quantification. All data were mean-centered and, finally, a calibration model was built by partial least-squares (PLS) regression (16). The optimal number of PLS factors was defined for which the root-mean-square error of crossvalidation (RMSECV) reached a minimum $(<1 \%$ of spectral variation). Cross-validation was conducted with the leave-oneout method; spectral preprocessing and PLS regression were calculated using Matlab ${ }^{\circledR}$ (MathWorks, Naticks, MA, USA). The calibration models were evaluated in terms of the correlation coefficient, $R^{2}$, the cross-validation coefficient, $Q^{2}$, the root-mean-square error of calibration (RMSEG), and of crossvalidation (RMSECV).

The concentration of solubilized drug in the digests was determined using an off-line method as reference for the calibration of Raman spectra (14). A $2 \mathrm{ml}$ sample was removed from the lipolysis medium prior to and after 5, 10, 15, 20, 25, 30, 40, 50, and $60 \mathrm{~min}$ of lipolysis. The samples were directly passed through an Anotop 25 Plus syringe filter (pore size $0.1 \mu \mathrm{m}$, aluminum oxide filter membrane) and the filtrate was immediately diluted in acetonitrile to halt further lipolysis. Control experiments were also performed that verified that no loss of dissolved drug occurred via potential filter adsorption. The samples were then centrifuged $(16,000 \times \mathrm{g}, 30 \mathrm{~min})$ and analyzed by HPLC (14).

\section{X-ray Powder Diffraction}

X-ray powder diffraction (XRPD) was used to determine the solid-state properties of fenofibrate precipitating during in vitro lipolysis. LBFs containing fenofibrate were digested for $60 \mathrm{~min}$ and, after enzyme inhibition, an aliquot was 
ultracentrifuged $\left(80,000 \times \mathrm{g}, 37^{\circ} \mathrm{C}, 90 \mathrm{~min}\right)$ in a Centrikon T-1180 ultracentrifuge equipped with a TFT-80.4 fixedangle rotor (Kontron Instruments, Milan, Italy). The pellet phase was immediately isolated and an X-ray diffractogram was recorded. The same procedure was followed with the pellet phase obtained from a $60 \mathrm{~min}$ lipolysis experiment with drug-free formulation, which was spiked with an equal amount of pure fenofibrate (as used for LBF preparation). Moreover, the X-ray diffractogram of crystalline fenofibrate was recorded as reference. A theta-theta X-ray powder diffractometer (R-XRD Phaser D2, Bruker AXS GmbH, Karlsruhe, Germany) equipped with a Co and Cu KFL tube $(30 \mathrm{kV}, 10 \mathrm{~mA})$ as radiation source and a Lynxeye ${ }^{\circledR}$ detector was employed. The samples were scanned in the angular range of $5^{\circ}(2 \theta)$ to $40^{\circ}(2 \theta)$ with a step size of $0.1^{\circ}(2 \theta)$ and a count time of 5 s per step.

\section{Measurement of Fenofibrate Solubility in Lipolysis Medium at Different Lipolysis Times}

The solubility of fenofibrate in lipolysis medium containing $\mathrm{LBF}$ was determined at different lipolysis times $\left(C_{m, t}^{*}\right)$. In vitro lipolysis with drug-free formulation was performed as described and enzyme inhibitor (4-bromophenylboronic acid, $1 \mathrm{M}$ in methanol, $9 \mu \mathrm{l}$ per $\mathrm{ml}$ of digest) was added to the medium at defined time points to stop further digestion. An aliquot was transferred to hermetically sealed glass vials and excess solid fenofibrate was added. The samples were equilibrated $\left(450 \mathrm{rpm}, 37^{\circ} \mathrm{C}\right)$, and an aliquot was centrifuged after $4 \mathrm{~h}, 8 \mathrm{~h}$, and $24 \mathrm{~h}\left(16,000 \times \mathrm{g}, 37^{\circ} \mathrm{C}, 30 \mathrm{~min}\right)$. The solubilities were found to decrease after $24 \mathrm{~h}$ of equilibration, which was attributed to kinetic instability of the colloidal structures in digested medium $(14,17)$. The liquid phase was diluted in acetonitrile, centrifuged $(16,000 \times \mathrm{g}, 15 \mathrm{~min})$, and drug content analyzed by HPLC. Whenever a distinct lipid phase was obtained upon centrifugation, the lipid and the aqueous phase were re-emulsified to measure the overall concentration of solubilized drug. Drug solubility experiments were carried out in triplicate with each formulation.

\section{Determination of the In Vivo Bioavailability of Fenofibrate-Loaded LBFs}

The pharmacokinetic (PK) profiles of fenofibric acid after peroral (p.o.) administration of formulation IIIA LC, IIIA $\mathrm{MC}$, and IIIB/IV and after intravenous (i.v.) administration of a fenofibrate solution were obtained from the literature (15). Briefly, six male landrace pigs were fasted for $16 \mathrm{~h}$, after which the LBFs were administered in gelatine capsules with the aid of a dosing gun. The pigs received $\sim 50 \mathrm{ml}$ of tap water via a syringe, had continuous access to water during the study, and were fed $8 \mathrm{~h}$ after LBF administration. Blood samples were collected pre-dosing and 0.5, 1, 2, 3, 4, 6, 8, 12, 24, and
$48 \mathrm{~h}$ post-dosing. For the i.v. dosing, an ethanol-based solution containing $25 \mathrm{mg}$ fenofibrate was administered by slow infusion into an ear vein. Blood sampling was performed as outlined above, with additional blood samples taken at $0.0833,0.25,0.75 \mathrm{~h}$ post-dose. The concentration of the active metabolite fenofibric acid was then determined in blood samples, since fenofibrate is rapidly and completely transformed to fenofibric acid by tissue and plasma esterases (18).

\section{THEORETICAL SECTION}

A mathematical model that simulates the time course of drug supersaturation in the intestine after oral administration of LBFs was developed. This profile was then used to estimate the likelihood of lipolysis-triggered drug precipitation in an absorption environment. In order to model intestinal drug supersaturation, the concentration of solubilized drug and drug solubility in intestinal fluids was required. The intralumenal concentrations of solubilized drug were derived from in vivo plasma concentration versus time data. The time evolution of drug solubility in the intestinal fluids was calculated by combining in vitro solubility and lipolysis data with the pharmacokinetic parameters derived from the in vivo studies.

\section{Modeling the Drug Concentration Profile in the Intestine}

To model the profile of solubilized fenofibrate in the intestinal lumen, $C_{i d}(t)$, we fitted a physiologically based pharmacokinetic (PBPK) model to the in vivo plasma concentration data obtained from pig studies. The plasma profile of fenofibric acid obtained after i.v. administration was used to estimate the rate constants of drug disposition $\left(k_{p t}, k_{t p}\right)$ and elimination $\left(k_{e l}\right)$, as well as the volume of distribution in the plasma compartment $\left(V_{d}\right)$ by fitting to a two-compartment model to the experimental data according to the equations:

$\frac{d D_{p}(t)}{d t}=-k_{e l} \cdot D_{p}(t)-k_{p t} \cdot D_{p}(t)+k_{t p} \cdot D_{t}(t)$

$\frac{d D_{t}(t)}{d t}=k_{p t} \cdot D_{p}(t)-k_{t p} \cdot D_{t}(t)$

$C_{p}(t)=D_{p}(t) / V_{d}$

where $D_{p}$ and $C_{p}$ are the drug mass and concentration in the plasma, respectively; $D_{t}$ is the drug mass in the peripheral tissue; $k_{p t}$ and $k_{t p}$ are the rate constants for drug disposition from plasma to peripheral tissue and from peripheral tissue to plasma, respectively.The PK profiles obtained after oral 
administration of LBFs were then fitted using Eqs. 5-11

(Fig. 1):

$$
\begin{aligned}
& \frac{d D_{g u}(t)}{d t}=-k_{g d} \bullet D_{g u}(t)-k_{g e} \cdot D_{g u}(t) \\
& \frac{d D_{g d}(t)}{d t}=k_{g d} \bullet D_{g u}(t)-k_{g e} \bullet D_{g d}(t) \\
& \frac{d D_{i u}(t)}{d t}=k_{g e} \bullet D_{g u}(t)-k_{i d} \bullet D_{i u}(t)
\end{aligned}
$$

$$
\frac{d D_{i d}(t)}{d t}=k_{g e} \cdot D_{g d}(t)+k_{i d} \cdot D_{i u}(t)-P_{e f f, F F} \cdot \frac{2}{r} \cdot f \cdot D_{i d}(t) \cdot 3600
$$

$\frac{d D_{p}(t)}{d t}=P_{e f f, F F} \bullet \frac{2}{r} \cdot f \cdot D_{i d}(t) \cdot 3600-k_{e l} \bullet D_{p}(t)-k_{p t} \bullet D_{p}(t)+k_{t p} \bullet D_{t}(t)$

$C_{p}(t)=D_{p}(t) / V_{d} \bullet F_{a}$

$C_{i d}(t)=D_{i d}(t) / V_{i}$

$D_{g u}$ and $D_{g d}$ are the drug mass in undispersed and dispersed $\mathrm{LBF}$ in the stomach, respectively; $D_{i u}$ and $D_{i d}$ are the drug mass in undispersed and dispersed LBF in the intestine, respectively. We assumed an effective permeability coefficient for fenofibrate, $P_{e f f, F F}$, of $2.66 \cdot 10^{-5} \mathrm{~cm} / \mathrm{s}(13)$, an intestinal radius, $r$, of $1.5 \mathrm{~cm}(19)$, and an effective surface expansion factor, $f$, of 30 (20). A volume of intestinal fluids, $V_{i}$, of $50 \mathrm{ml}$ was assumed, which corresponded to the volume of co-administered water. The gastric emptying constant, $k_{g e}$, and the fraction absorbed, $F_{a}$, were estimated by regressing Eqs. 5-11 to the plasma concentration profiles obtained after p.o. administration of formulations IIIA LC, IIIA MC, and IIIB/IV.

The dispersion rate constants in gastric fluid, $k_{g d}$, and in intestinal fluid, $k_{i d}$, were obtained from the results of in vitro dissolution/dispersion testing reported by Griffin et al. (15). This test was performed using a mini-vessel dissolution apparatus containing fasted-state simulated gastric fluid (FaSSGF (21)) and fasted-state simulated intestinal fluid (FaSSIF-v2 (21)). The constants $k_{g d}$ and $k_{i d}$ were obtained by fitting a first-order kinetic equation to the dispersion profiles measured in FaSSGF and FaSSIF-v2, respectively (Table II).

It should be noted that this biopharmaceutical model assumed no drug precipitation in the GI lumen. Moreover, the model assumptions included that drug absorption occurred only in the intestine and only from dispersed formulation (i.e., no absorption occurred in the stomach and from undispersed formulation). Modeling was performed using Matlab. The differential equations were numerically solved using the ode 45 solver, and optimization procedures were carried out with the fminsearch algorithm.
To evaluate the goodness of fit (modeled versus experimental plasma data), the similarity factor, $f_{2}$, was calculated as follows:

$f_{2}=50 \cdot \log \left(\sqrt{1+\frac{1}{n} \sum_{i=1}^{n}\left(M_{i}-T_{i}\right)^{2}} \cdot 100\right)$

where $n$ is the number of sampling time points, $M_{i}$ is the ratio between the plasma concentration at time point $i$ and the maximum plasma concentration in the modeled profile, and $T_{i}$ is the ratio between the plasma concentration at time point $i$ and the maximum value in the experimental plasma concentration profile (22).

\section{Modeling the Time Evolution of Drug Solubility During LBF Digestion}

A mathematical approach to model drug solubility during formulation lipolysis in the intestinal environment was developed. Initially, a general model of drug solubility during in vitro formulation lipolysis was developed. Subsequently, this model was applied to simulate drug solubility in the intestinal environment (i.e., under continuous absorptive conditions). This solubility profile is finally used to calculate the time course of drug supersaturation and, hence, to estimate the likelihood of precipitation from LBFs under physiological conditions.

\section{Theoretical Concept}

Previously, we have reported a heuristic approach to model the time evolution of drug solubility during in vitro lipolysis (14). This approach assumed that different lipidic species contributed additively to the overall drug solubility. Therefore, drug solubility was proportional to the concentration of lipids and of lipolysis products (LPs). A similar assumption was applied to the current study to model the time evolution of drug solubility during LBF digestion. However, the model was intended to simulate drug solubility not only during in vitro lipolysis (closed system), but also during the intestinal transit of LBFs (open system). Some adaptations of the previous mathematical model were therefore necessary.

The theoretical concept of solubility modeling is represented graphically in Fig. 2. The amount of TGs and SFs decreases during formulation digestion. As it can be assumed that there is linearity between the concentration of lipids and drug solubility $(23,24)$, the drug solubilization caused by these excipients reduces accordingly $\left(C_{T G, t}^{*}\right.$ and $C_{S F, t}^{*}$ respectively). At the same time, the hydrolysis of TGs and SFs liberates LPs, which can contribute to drug solubilization. This increasing solubility is represented by $C_{L P 1, t}^{*}$ and $C_{L P 2, t}^{*}$, where LP1 are lipolysis products liberated from TGs and LP2 are lipolysis products liberated from SFs. Fenofibrate solubility in pure 


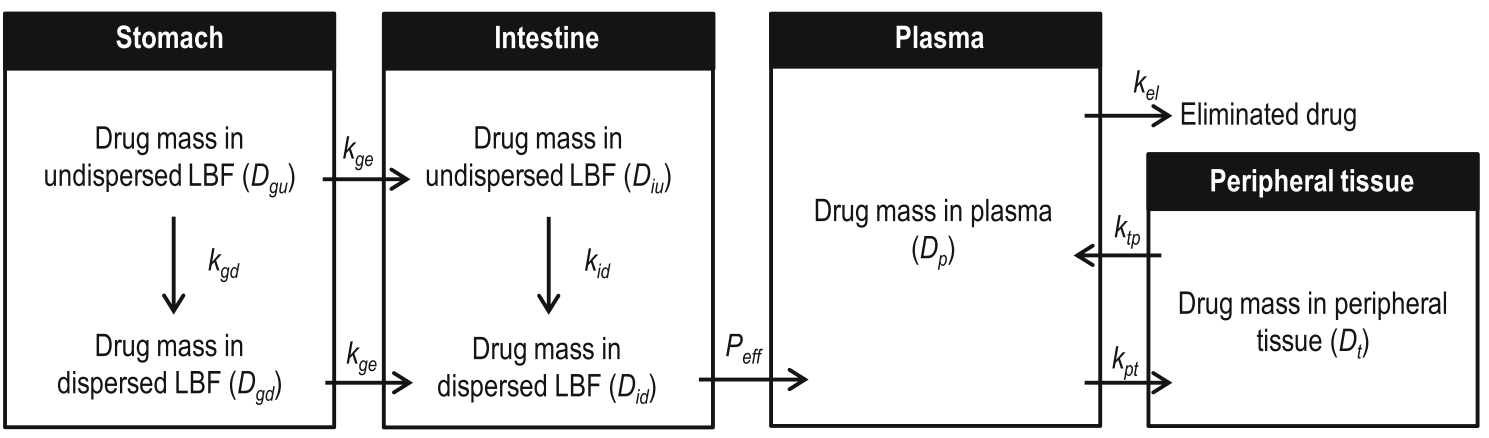

Fig. I Scheme of model parameters describing drug transit after oral administration of LBFs.

lipolysis medium (without LBF) was comparatively small and was hence considered negligible for solubility modeling.

In summary, the overall drug solubility in the lipolysis medium, $C_{m, t}^{*}$, was calculated by considering the contribution of each lipidic species additively:

$C_{m, t}^{*}=C_{T G, t}^{*}+C_{S F, t}^{*}+C_{L P 1, t}^{*}+C_{L P 2, t}^{*}$

To determine these contributions, a relative drug solubility value was introduced, which was the amount of drug that is solubilized by $1 \mathrm{mmol}$ of excipient per $\mathrm{ml}$ of lipolysis medium at equilibrium $\left(\mathrm{mg} \cdot \mathrm{ml}^{-1} \cdot \mathrm{mmol}^{-1}\right)$. Thus, the product of the relative fenofibrate solubility $\left(\mathrm{mg} \cdot \mathrm{ml}^{-1}\right)$ and the corresponding amount of excipient as a function of time (mmol) determined the time course of each partial contribution to drug solubility.

$C_{m}^{*}(t)=\hat{C}_{T G}^{*} \bullet T G(t)+\hat{C}_{S F}^{*} \bullet S F(t)+\hat{C}_{L P 1}^{*} \bullet L P 1(t)+\hat{C}_{L P 2}^{*} \bullet L P 2(t)$

where $\hat{C}_{T G}^{*}, \hat{C}_{S F}^{*}, \hat{C}_{L P 1}^{*}$, and $\hat{C}_{L P 2}^{*}$ are the relative fenofibrate solubilities in TGs, SFs, LP1, and LP2, respectively, and $T G(t), S F(t), L P 1(t)$, and $L P 2(t)$ are the molar amounts of excipient at time $t$. Formulation IIIB/IV contained SFs only and, thus, the overall drug solubility was calculated according to:

$C_{m}^{*}(t)=\hat{C}_{S F}^{*} \cdot S F(t)+\hat{C}_{L P 2}^{*} \bullet L P 2(t)$

Table I Composition of the formulations IIIA LC, IIIA MC, and IIIB/IV, according to the lipid-based formulation classification system (LFCS)

\begin{tabular}{llll}
\hline Component & IIIA LC & IIIA MC & IIIB/V \\
\hline Olive oil & $40 \% \mathrm{w} / \mathrm{w}$ & - & - \\
Miglyol 8I2 & - & $40 \% \mathrm{w} / \mathrm{w}$ & - \\
Cremophor RH 40 & $20 \% \mathrm{w} / \mathrm{w}$ & $20 \% \mathrm{w} / \mathrm{w}$ & $33 \% \mathrm{w} / \mathrm{w}$ \\
Tween 85 & $40 \% \mathrm{w} / \mathrm{w}$ & $40 \% \mathrm{w} / \mathrm{w}$ & $67 \% \mathrm{w} / \mathrm{w}$ \\
Fenofibrate & $80 \mathrm{mg} / \mathrm{g}$ & $80 \mathrm{mg} / \mathrm{g}$ & $80 \mathrm{mg} / \mathrm{g}$ \\
\hline
\end{tabular}

The values of $\hat{C}_{T G}^{*}, \hat{C}_{S F}^{*}, \hat{C}_{L P 1}^{*}$, and $\hat{C}_{L P 2}^{*}$ were derived from experimental solubility data as described in the next section. Modeling and simulation of solubility profiles was performed using Matlab.

\section{Calculation of Relative Drug Solubilities}

The relative drug solubilities $\hat{C}_{T G}^{*}, \hat{C}_{S F}^{*}, \hat{C}_{L P 1}^{*}$, and $\hat{C}_{L P 2}^{*}$ were obtained from experimental solubility values with undigested (for $\hat{C}_{T G}^{*}$ and $\hat{C}_{S F}^{*}$ ) and digested (for $\hat{C}_{L P 1}^{*}$, and $\hat{C}_{L P 2}^{*}$ ) formulations.

Formulation IIIB/IV was composed of SFs only, and hence, the drug solubilities measured in undigested and digested formulation IIIB/IV were used to calculate $\hat{C}_{S F}^{*}$ and $\hat{C}_{L P 2}^{*}$, respectively. $\hat{C}_{S F}^{*}$ corresponded to the solubility $C_{m, 0}^{*}$ (i.e., drug solubility in $100 \%$ undigested SF) divided by the molar amount of SF in dispersion at $t=0, S F_{\text {tot }}$ (Eq. 18). $\hat{C}_{L P 2}^{*}$ was the relative drug solubility in completely digested formulation IIIB/IV (i.e., only LP2). An experimental value was not available, because formulation IIIB/IV was only partially digested by $60 \mathrm{~min}$ of in vitro lipolysis. However, it is known from literature that there is a linear relationship between the concentration of a SF and drug solubility (23). Thus, $\hat{C}_{L P 2}^{*}$ was determined via linear extrapolation based on the measured drug solubility, $C_{m, 60}^{*}$, and the extent of digestion by $60 \mathrm{~min}$ of lipolysis; the resulting value was then divided by the molar amount of LP2, $L P 2_{\text {tot }}$ (Eq. 17). It should be noted that

Table II Dispersion rate constants (value $\pm 95 \% \mathrm{Cl}$ ) in FaSSGF and FaSSIF$\mathrm{v} 2$, as obtained from the fitting of data described in the literature (I5). The distinction between dispersed an undispersed formulation was given by the experimental mini-vessel test. The dispersion rates were described excellently by a first order kinetic equation $\left(R^{2}>0.93\right)$

\begin{tabular}{lrr}
\hline Formulation & $k_{g d}\left(h^{-1}\right)$ & \multicolumn{1}{c}{$k_{\text {id }}\left(h^{-1}\right)$} \\
\hline IIIA LC & $10.9 \pm 1.3$ & $15.2 \pm 1.5$ \\
IIIA MC & $7.4 \pm 3.1$ & $7.2 \pm 1.9$ \\
IIIB/IV & $9.0 \pm 1.8$ & $6.4 \pm 0.6$ \\
\hline
\end{tabular}


Fig. 2 Time evolution of (a) lipidic species and (b) drug solubilities in the lipolysis medium during LBF digestion (assuming no LPs absorption). Colored lines represent the amounts and solubility contributions of the single lipidic species and the black line represents the resulting overall drug solubility
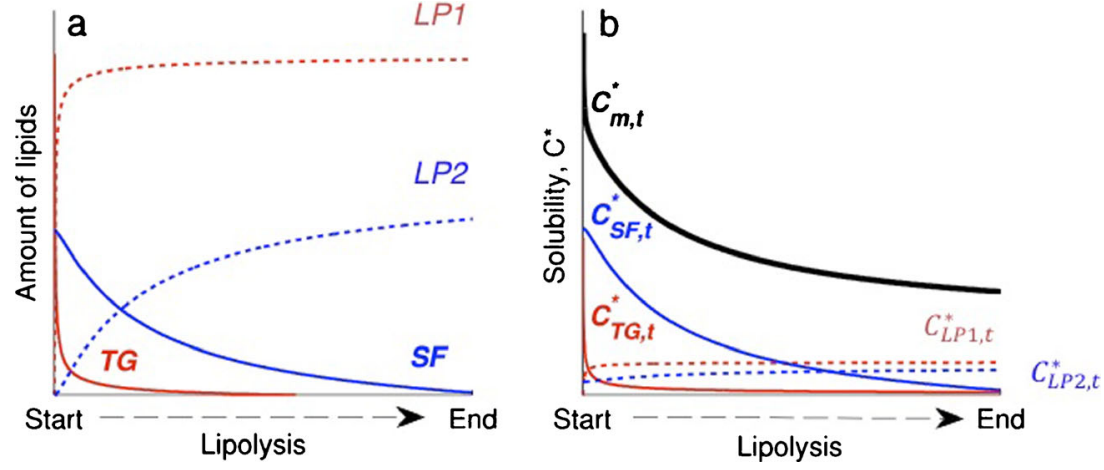

formulation IIIA LC and IIIA MC also contained Cremophor RH 40 and Tween 85 at a ratio of 1:2. Thus, the calculated values of $\hat{C}_{S F}^{*}$ and $\hat{C}_{L P 2}^{*}$ were the same for each formulation.

The relative solubility $\hat{C}_{T G}^{*}$ was calculated for Type IIIA LC and IIIA MC only. Prior to lipolysis, fenofibrate was solubilized by undigested SFs and TGs. Thus, the contribution of TGs to drug solubility was the difference between the overall drug solubility, $C_{m, 0}^{*}$, and the contribution of SFs, $C_{S F}^{*} C_{S F}^{*}$ was the product of the relative solubility, $\hat{C}_{S F}^{*}$, and the molar amount of undigested SF prior to lipolysis $\left(S F_{t o t}\right)$. This difference was then divided by the molar amount of TGs in dispersion at $t=0, T G_{t o t}$ (Eq. 18).

$\hat{C}_{S F}^{*}=C_{m, 0}^{*} / S F_{t o t}$

$\hat{C}_{L P 2}^{*}=\left(C_{m, 0}^{*}-\frac{C_{m, 0}^{*}-C_{m, 60}^{*}}{\text { Extent of digestion }}\right) / L P 2_{\text {tot }}$

$\hat{C}_{T G}^{*}=\left(C_{m, 0}^{*}-\hat{C}_{S F}^{*} \cdot S F_{t o t}\right) / T G_{t o t}$

Finally, $\hat{C}_{L P 1}^{*}$ was obtained by regressing Eq. 14 to the experimental solubility values, $C_{m, t}^{*}$, for formulations IIIA LC and IIIA MC.

\section{Determination of the Kinetics of TG and SF Digestion}

The kinetics of formulation digestion was determined using in vitro lipolysis data. First, the course of TG and SF digestion was determined from the FA titration profiles by considering the stoichiometry of hydrolysis. It was assumed that TGs were digested rapidly on lipolysis initiation, whereas SFs were digested more slowly in a second stage of lipolysis. This twostep lipolysis kinetics arises from the different hydrolysis rates of lipolytic enzymes present in the pancreatic extract. The pancreatic lipase and co-lipase preferentially bind insoluble lipids and, therefore, they hydrolyze primarily TGs and DGs. In contrast, the carboxyl ester hydrolase shows higher affinity to lamellar and micellar structures containing SFs $(25,26)$. Moreover, the lipolytic activity of the pancreatic lipase and co- lipase is higher compared to that of the cholesterol ester hydrolase $(25,26)$. For formulation IIIA LC and IIIA MC, two time profiles were anticipated: one for the digested TGs (first stage) and one for the digested SFs (second stage). Instead, only one stage of lipolysis was assumed for the surfactant-only formulation IIIB/IV. Based on the resulting profiles of undigested glycerides ( $\mathrm{mmol}$ ) versus time, we defined a general power-law describing the digestion rate as a function of the amount of available substrate:

$\frac{d T G(t)}{d t}=-k_{d i g, T G} \cdot(T G)^{n_{T G}}$

$\frac{d S F(t)}{d t}=-k_{d i g, S F} \bullet(S F)^{n_{S F}}$

where $k_{d i g, T G}$ and $k_{d i g, S F}$ are the digestion rate constants, and $n_{T G}$ and $n_{S F}$ are the order of kinetics for TG and SF digestion, respectively. These constants were estimated by regressing Eqs. 19 and 20 to the experimental values.

\section{General Model of Formulation Digestion Used for Solubility Modeling}

To describe the kinetics of LBF digestion with a timeindependent transition from the first to the second stage of hydrolysis, formulation digestion was modeled using Eqs. 2124. According to the two-step kinetics, SFs were expected to be digested after forming micelles. The kinetics of micelles formation was assumed to correspond to the kinetics of TG digestion. Hence, Eqs. 22 and 23 describe the time evolution of SFs in oil droplets and in micelles, respectively. The total amount of undigested SFs, which is ultimately used for solubility modeling, is described by Eq. 24. The generation of LP1 (Eq. 25) and LP2 (Eq. 26) corresponded to the kinetics of TG and SF digestion, respectively, according to the stoichiometry of lipolysis.

$\frac{d T G(t)}{d t}=-k_{d i g, T G} \bullet(T G(t))^{n_{T G}}$ 
$\frac{d S F_{\text {oil }}(t)}{d t}=-k_{d i g, T G} \bullet(T G(t))^{n_{T G}} \cdot \frac{\mathcal{N}_{S F}}{\mathcal{N}_{T G}}$

$\frac{d S F_{\text {mic }}(t)}{d t}=k_{d i g, T G} \bullet(T G(t))^{n_{T G}} \cdot \frac{\mathcal{N}_{S F}}{\mathcal{N}_{T G}}-k_{d i g, S F} \bullet\left(S F_{\text {mic }}(t)\right)^{n_{S F}}$

$\frac{d S F(t)}{d t}=-k_{d i g, S F} \bullet\left(S F_{\text {mic }}(t)\right)^{n_{S F}}$

$\frac{d L P 1(t)}{d t}=k_{d i g, T G} \bullet(T G(t))^{n_{T G}}$

$\frac{d L P 2(t)}{d t}=k_{d i g, S F} \bullet\left(S F_{i d, m i c}(t)\right)^{n_{S F}}$

$\mathcal{N}_{S F} / \mathcal{N}_{T G}$ is the ratio of molar amounts of SFs and TGs in undigested formulation.To model the lipolysis-triggered change in drug solubility during in vitro lipolysis (closed system), the numerical solutions of Eqs. 21, 24, 25, and 26 was inserted into Eqs. 14 and 15.

\section{Modeling Intestinal Formulation Digestion and Drug Solubility}

Intestinal formulation lipolysis and drug solubility were simulated using the general model of formulation digestion (Eqs. 21-24) and the physiological parameters describing the GI transit of LBFs. As a first approximation, it was assumed that (i) there is no lipolysis in the stomach; (ii) TGs and DGs are not absorbed from the GI tract; (iii) LPs are absorbed only in the intestine. The kinetics of undigested lipid-based excipients in the GI tract can be described as follows:

$\frac{d X_{g u}(t)}{d t}=-k_{g d} \cdot X_{g u}(t)-k_{g e} \cdot X_{g u}(t)$

$\frac{d X_{g d}(t)}{d t}=k_{g d} \cdot X_{g u}(t)-k_{g e} \cdot X_{g d}(t)$

$\frac{d X_{i u}(t)}{d t}=k_{g e} \cdot X_{g u}(t)-k_{i d} \bullet X_{i u}(t)$

where $X$ holds for the amount of TGs or SFs; $X_{g u}$ and $X_{g d}$ are the amounts of excipient in the stomach in undispersed and dispersed formulation, respectively, and $X_{i u}$ is the amount of undispersed excipients in the intestine. The time evolution of dispersed TGs and SFs in the intestine depends on the lipolysis rate of the specific glyceride and was described by the following equations: $\frac{d T G_{i d}(t)}{d t}=k_{g e} \bullet T G_{g d}(t)+k_{i d} \bullet T G_{i u}(t)-k_{d i g, T G} \bullet\left(T G_{i d}(t)\right)^{n_{T G}}$

$\frac{d S F_{i d, o i l}(t)}{d t}=k_{g e} \bullet S F_{g d, o i l}(t)+k_{i d} \bullet S F_{\text {iu,oil }}(t)-k_{d i g, T G} \bullet(T G(t))^{n_{T G}} \bullet \frac{\mathcal{N}_{S F}}{\mathcal{N}_{T G}}$

$\frac{d S F_{i d, m i c}(t)}{d t}=k_{d i g, T G} \bullet(T G(t))^{n T G} \cdot \frac{\mathcal{N}_{S F}}{\mathcal{N}_{T G}}-k_{d i g, S F} \bullet\left(S F_{i d, m i c}(t)\right)^{n_{S F}}$

$\frac{d S F_{i d}(t)}{d t}=k_{g e} \bullet S F_{g d}(t)+k_{i d} \bullet S F_{i u}(t)-k_{d i g, S F} \bullet\left(S F_{i d, m i c}(t)\right)^{n_{S F}}$

$\frac{d L P 1(t)}{d t}=k_{d i g, T G} \bullet\left(T G_{i d}(t)\right)^{n_{T G}}-P_{e f f, L P} \bullet \frac{2}{r} \bullet f \bullet L P 1(t) \bullet 3600$

$\frac{d L P 2(t)}{d t}=k_{d i g, S F} \bullet\left(S F_{i d, m i c}(t)\right)^{n_{S F}}-P_{e f f, L P} \bullet \frac{2}{r} \cdot f \cdot L P 2(t) \bullet 3600$

Eq. 30 denotes the time course of undigested TGs and Eqs. 31 and 32 describe the time evolution of undigested SFs in oil droplets and in micelles, respectively. The time evolution of LP1 is determined by the lipolysis kinetics of TGs and the absorption rate of LPs (Eq. 34), and that of LP2 is given by the lipolysis kinetics of SFs and the absorption rate of LPs (Eq. 35). $P_{e f f, L P}$ is the effective permeability of LPs and the same value was assumed for LP1 and LP2.

Drug solubility in the intestine was finally modeled by inserting the numerical solutions of Eqs. 30, 33, 34, and 35 into Eqs. 14 and 15. Only intestinal drug solubility was simulated, because the intestine is the primary site of formulation lipolysis. The GI transit of excipients after p.o. administration of the LBFs is visualized in Fig. 3.

\section{Calculation of the Saturation Ratio During LBF Digestion}

The drug saturation ratio, $S R(t)$, during formulation lipolysis was calculated according to:

$S R(t)=\frac{C(t)}{C^{*}(t)}$

where $C(t)$ is the actual concentration of solubilized drug and $C^{*}(t)$ the drug solubility. $S R(t)$ during in vitro lipolysis was calculated using the $C(t)$ obtained from Raman spectroscopy, whereas $S R(t)$ in the in vivo situation was calculated using the $C(t)$ values obtained from Eq. 11. For in vitro lipolysis, the 
recently proposed maximum supersaturation ratio, $S R^{M}$, was calculated according to (27):

$S R^{M}=\frac{C_{\max }}{C_{m, 60}^{*}}$

where $C_{\max }$ is the maximum attainable concentration of fenofibrate in solution assuming no precipitation and $C_{m, 60}^{*}$ is the lowest fenofibrate solubility in the lipolysis medium during digestion ( $t=60 \mathrm{~min})$.

\section{Parameter Sensitivity Analysis}

The impact of the LP absorption rate on intralumenal drug solubility and supersaturation was studied. Thus, a $P_{\text {eff }, L P}$ of 0 , $10^{-7}, 10^{-5}$, and $10^{-3} \mathrm{~cm} / \mathrm{s}$ was inserted into Eqs. 34 and 35 to simulate no absorption as well as slow, moderate, and fast LP absorption, respectively. The resulting drug solubility and supersaturation profiles were analyzed.

Moreover, intestinal drug supersaturation profiles were simulated for different drug permeability values, $P_{e f f,}$ and lipolysis rates. $P_{\text {eff } F F}$ was varied in the range of 0.1 to 10 times the nominal value of $2.66 \cdot 10^{-5} \mathrm{~cm} / \mathrm{s}$ and the lipolysis rate was varied in the range of 0.01 to 100 times the lipolysis rates obtained from in vitro experiments. The result was depicted as a 3D surface plot showing the peak saturation ratio as a function of $P_{e f f}$ and relative lipolysis rate.

\section{RESULTS}

\section{Rate and Extent of LBF Digestion}

The digestion of three LBFs was studied using the in vitro lipolysis method (8). The amount of unionized FAs was measured via back-titration and the resulting correction factors were $1.61,1.10$, and 1.30 for formulation IIIA LC, IIIA MC, and IIIB/IV, respectively. Hence, the largest fraction of unionized FAs was measured with long-chain FAs $(61 \%$ in formulation IIIA LC and 30\% in formulation IIIB/IV). This result was a consequence of the higher $\mathrm{pK}_{\mathrm{a}}$ of long-chain FAs compared to medium-chain FAs and is in agreement with previously reported values (28).

Different rates and extents of digestion were observed among the three formulations (Fig. 4). A two-stage profile resulted from the digestion of IIIA LC and IIIA MC, consisting of an initial stage of rapid lipolysis followed by a second stage of slower lipolysis. These two formulations were completely hydrolyzed within $60 \mathrm{~min}$ with an extent of digestion of $109.4 \pm 5.9 \%$ and $103.3 \pm 0.03 \%$ (mean $\pm 1 \mathrm{SD}, n=3$ ), respectively. Lipolysis of formulation IIIB/IV was comparatively slow and only $56.0 \pm 2.0 \%$ of excipients were digested. The two-stage lipolysis profile of formulations IIIA LC and
IIIA MC agreed with our assumption of fast TG and slow SF digestion. Analogously, only one stage of lipolysis was observed with formulation IIIB/IV, due to the presence of only SFs and the absence of an oil phase.

The amount of digested glycerides was calculated based on the titration profiles in Fig. 4 and the stoichiometry of lipolysis. The rate of TG and SF lipolysis was then determined according to Eqs. 19 and 20, and the resulting kinetic constants $k_{d i g, T G}, k_{d i g, S F}, n_{T G}$, and $n_{S F}$ are detailed in Table III. The power-laws described the profiles of glyceride digestion accurately, resulting in $R^{2}>0.92$.

\section{Determination of Lipolysis-Triggered Drug Precipitation (in vitro)}

The three formulations containing $80 \mathrm{mg} / \mathrm{g}$ fenofibrate were digested in vitro and the course of drug precipitation was monitored using in-line Raman spectroscopy. A specific calibration model was built for each formulation based on a set of 30 reference spectra spanning the entire concentration range of precipitated drug. Accurate calibration models were obtained with $R^{2}$ values of 0.9862 to 0.9974 and a RMSEC between 0.0124 and $0.0046 \mathrm{mg} / \mathrm{ml}$. The cross-validation procedure yielded $Q^{2}$ values of 0.9244 to 0.9561 and RMSECV smaller than $0.16 \mathrm{mg} / \mathrm{ml}$, indicating robust calibrations.

These PLS models were then applied to all Raman spectra of the corresponding LBF to determine the drug solubilization profiles over the $60 \mathrm{~min}$ digestion period. As a result, each LBF demonstrated extensive drug precipitation as seen in Fig. 5, showing the kinetics of solubilized drug. With formulations IIIA LC and IIIB/IV, fenofibrate started to precipitate after an initial lag phase of 10 to $15 \mathrm{~min}$. In contrast, with formulation IIIA MC, drug precipitation started rapidly after lipolysis initiation and reached equilibrium after $\sim 10 \mathrm{~min}$.

Interestingly, Griffin et al. (15) reported notably different precipitation profiles. Only formulation IIIB/IV resulted in comparable extent of drug precipitation $(\sim 70 \%)$, whereas less than $10 \%$ of the drug precipitated with IIIA LC and IIIA MC. These differences in the extent of precipitation between the two studies were most likely due to the different drug contents in the lipolysis assay (80 $\mathrm{mg}$ versus $240 \mathrm{mg}$ ), different amounts of formulation lipid ( $1 \mathrm{~g}$ versus $3 \mathrm{~g}$ ), and a minor difference in digestion media (i.e., type of bile salts).

To study the solid-state of precipitated drug, a pellet was isolated after ultracentrifugation at the end of the in vitro lipolysis experiment and then analyzed by XRPD. Figure 6 depicts the resulting XRPD spectra of the pellet phases obtained by ultracentrifugation, of pure crystalline fenofibrate, and of a reference pellet. This reference was the pellet obtained after lipolysis of drug-free formulation, which was spiked with crystalline fenofibrate. The angular range of peaks of the pellets containing precipitated drug corresponded to that of the reference pellet. Therefore, XRD analyses suggested the 


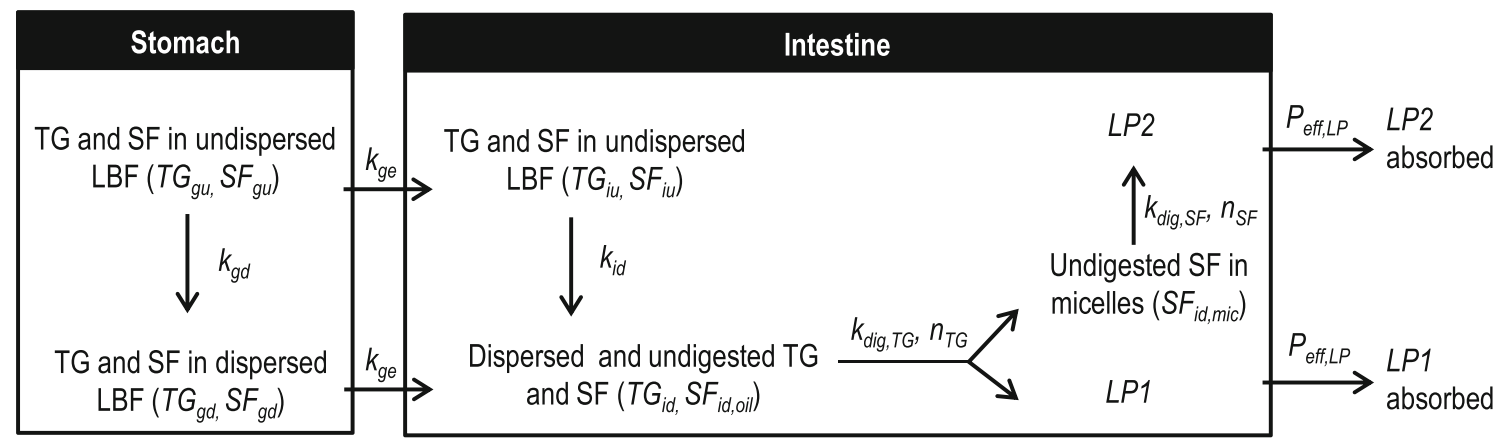

Fig. 3 Scheme of model parameters describing the Gl transit of excipients after oral administration. The distinction between dispersed and undispersed formulation was given by the experimental mini-vessel test in (I5).

precipitation of fenofibrate in a crystalline state during lipolysis with each formulation. This observation was in good agreement with Raman spectroscopy data, which showed a clear shift of fenofibrate peaks toward the position of crystalline reference material.

\section{Drug Solubility During in Vitro Lipolysis}

Fenofibrate solubility in lipolysis medium, $C_{m, t}^{*}$, was determined at different digestion times (Fig. 7). The highest initial solubility, $C_{m, 0}^{*}$, was obtained with formulation IIIA MC (2.19 $\pm 0.01 \mathrm{mg} / \mathrm{ml}$, mean $\pm \mathrm{SD}, n=3)$, followed by IIIA LC $(1.41 \pm$ $0.03 \mathrm{mg} / \mathrm{ml})$ and IIIB/IV $(0.93 \pm 0.01 \mathrm{mg} / \mathrm{ml})$. On lipolysis initiation, the solubilities decreased in each case due to the generation of comparatively hydrophilic lipolysis products. This decrease in drug solubility was most extensive with IIIA MC, where $80 \%$ lower solubility was measured after 5 min of digestion $\left(C_{m, 5}^{*}: 0.44 \pm 0.01 \mathrm{mg} / \mathrm{ml}\right)$, and less pronounced with IIIA LC and IIIB/IV, which resulted in a $C_{m, 5}^{*}$ of $0.81 \pm 0.03$ and $0.67 \pm 0.07 \mathrm{mg} / \mathrm{ml}$, respectively. By $60 \mathrm{~min}$ of digestion, the total loss of solubilization capacity was $72.3 \%, 87.6 \%$, and $50.0 \%$ for formulation IIIA LG, IIIA MC, and IIIB/IV, respectively.

The relative fenofibrate solubilities $\hat{C}_{T G}^{*}, \hat{C}_{S F}^{*}, \hat{C}_{L P 1}^{*}$, and $\hat{C}_{L P 2}^{*}$ were calculated based on measured drug solubility values and the molar amounts of excipients in the LBFs. As seen in Table IV, the relative fenofibrate solubility in long-chain TGs was lower than in medium-chain TGs (0.631 versus $0.701 \mathrm{mg}$. $\left.\mathrm{ml}^{-1} \cdot \mathrm{mmol}^{-1}\right)$. In contrast, drug solubility in long-chain LPs was higher than in medium-chain LPs $\left(0.369\right.$ versus $0.093 \mathrm{mg} \cdot \mathrm{ml}^{-1}$. $\left.\mathrm{mmol}^{-1}\right)$. To calculate $\hat{C}_{S F}^{*}$ and $\hat{C}_{L P 2}^{*}$ no differentiation was done between Cremophor RH 40 and Tween 85.

\section{Modeling Drug Solubility and Supersaturation During in Vitro Lipolysis}

The drug solubility as a function of time during in vitro lipolysis (without absorption sink) was modeled initially, given that these experimentally determined solubility values were available for model validation. Figure 7 displays the experimental (circles) and modeled (continuous lines) drug solubility profiles. There were excellent correlations between experimental and modeled profiles $\left(R^{2}>0.89\right)$.

The $S R$ s during in vitro lipolysis were calculated according to Eq. 36 based on the concentrations of solubilized drug obtained from Raman spectroscopy and the modeled drug solubility profiles. Prior to lipolysis, dispersed formulations IIIA LG and IIIB/IV were slightly supersaturated ( $S R$ of 1.36 and 1.94, respectively). In contrast, IIIA MC was initially below saturation $S R$ of 0.83 ) but rapidly became supersaturated on lipolysis initiation. The $S R$ s of all three formulations increased following initiation of lipolysis reaching a peak $S R$ value of $2.62,3.34$, and 2.31 with formulations IIIA LG, IIIA $\mathrm{MC}$, and IIIB/IV, respectively.

For comparison, the $S R^{M}$ values were also calculated, which is the ratio of the highest drug concentration in solution to the lowest drug solubility (i.e., $C_{m, 60}^{*}$ ). This theoretical value has been previously defined to indicate the maximum $S R$ obtainable during in vitro lipolysis (27). The values were generally higher than the $S R$ and the formulation rank order was slightly different, i.e., IIIA MC (6.81)> IIIB/IV (3.70)> IIIA LG (3.17).

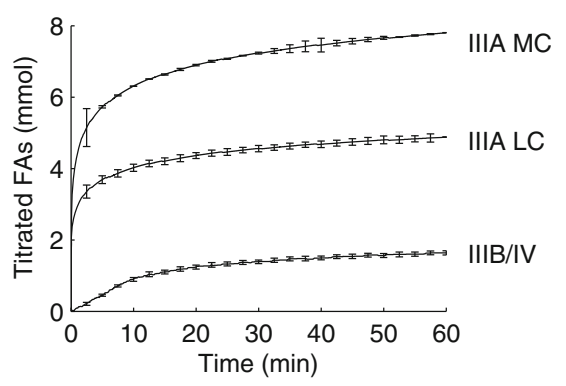

Fig. 4 Digestion profiles of the three model LBFs containing $80 \mathrm{mg} / \mathrm{g}$ fenofibrate (in vitro lipolysis according to Sek et al. (8)). The $\mathrm{NaOH}$ volume obtained from direct titration was adjusted for the amount of protonated FAs at $\mathrm{pH} 7.5$ (via back-titration) and the $\mathrm{NaOH}$ consumption caused by the digestion of phospholipids was subtracted from the total volume (mean \pm I $\mathrm{SD}, n=3)$. 
Table III Kinetic constants (value $\pm 95 \% \mathrm{Cl}$ ) for glyceride digestion

\begin{tabular}{|c|c|c|c|c|c|c|}
\hline \multirow[t]{2}{*}{$\angle B F$} & \multicolumn{3}{|l|}{ TG digestion } & \multicolumn{3}{|l|}{ SF digestion } \\
\hline & $k_{\text {dig, TG }}$ & $n_{T G}$ & $R^{2}$ & $k_{\text {dig,SF }}$ & $n_{S F}$ & $R^{2}$ \\
\hline IIIA LC & $587.7 \pm 95.9$ & $1.35 \pm 0.18$ & 0.9628 & $25.93 \pm 0.55$ & $2.17 \pm 0.04$ & 0.9937 \\
\hline IIIA MC & $276.5 \pm 13.6$ & $2.30 \pm 0.15$ & 0.9768 & $18.08 \pm 0.36$ & $1.29 \pm 0.03$ & 0.9851 \\
\hline IIIB/IV & - & - & - & $1.18 \pm 0.03$ & $5.32 \pm 0.06$ & 0.9216 \\
\hline
\end{tabular}

\section{Modeling Lipolysis-Triggered Drug Supersaturation in the Intestinal Lumen}

\section{In Vivo LBF Performance and Estimation of PK Parameters}

Griffin et al. (15) previously reported absolute oral bioavailabilities of $65.6 \%, 70.7 \%$, and $71.7 \%$ for formulation IIIA LC, IIIA MC, IIIB/IV, respectively. These relatively high and
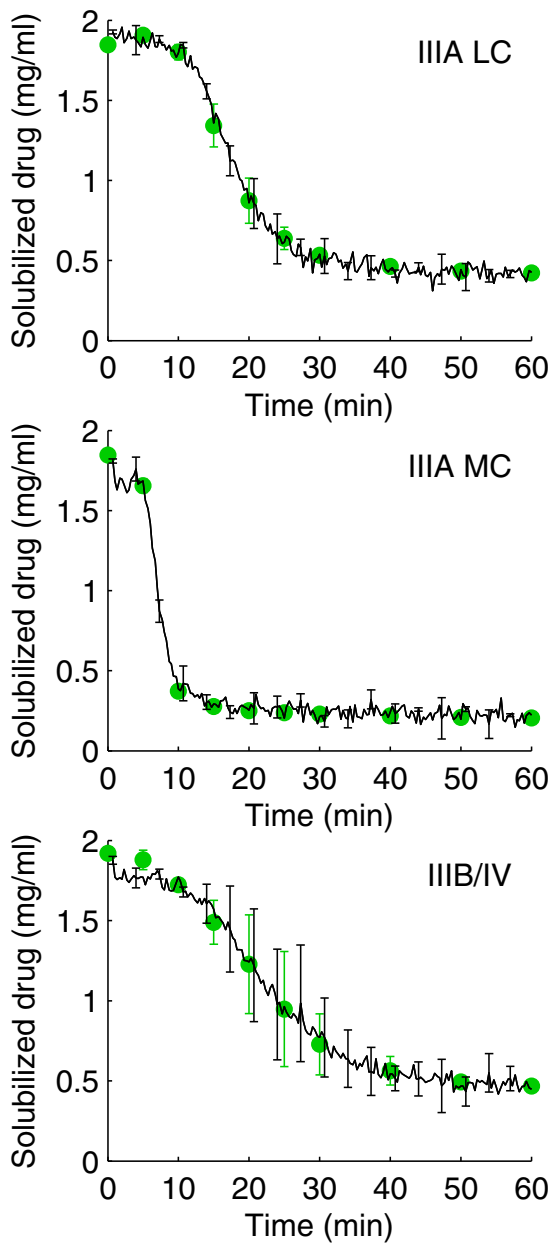

Fig. 5 Drug solubilization profiles measured with in-line Raman spectroscopy during in vitro lipolysis of LBF IIIA LC, IIIA MC, and IIIB/N. Decreasing concentrations of solubilized drug indicated precipitation. The black line represent the concentration profiles determined via Raman spectroscopy (mean $\pm \mid \mathrm{SD}, n=3$ ) and green circles are reference values measured upon nanofiltration (mean \pm I SD, $n=3$ ). similar oral bioavailabilities suggested that intralumenal fenofibrate precipitation was either absent or only minimal and similar for the three formulations. The in vivo data were used to estimate the PK parameters as well as the intralumenal drug concentration profiles of fenofibrate.

The plasma concentrations obtained after i.v. administration of fenofibrate allowed an estimation of $V_{d}, k_{e l}, k_{p t}$, and $k_{t p}$ and the resulting parameter values were $6.4 \pm 0.2 \mathrm{~L}, 0.187 \pm$ $0.008 \mathrm{~h}^{-1}, 0.093 \pm 0.046 \mathrm{~h}^{-1}$, and $0.179 \pm 0.025 \mathrm{~h}^{-1}$, respectively $\left(R^{2}=0.9963\right.$, Fig. 8). The similarity factor $f_{2}$ of 61.1 indicated that the difference between modeled and experimental plasma concentration profiles was less than $10 \%$.

The plasma concentrations obtained after p.o. administration of fenofibrate were then modeled using Eqs. 5-11. This model assumed that the entire oral dose was available for absorption, i.e., that no precipitation occurred in the GI lumen. As seen in Fig. 8, the plasma profiles of fenofibric acid were described accurately by the mathematical model. The similarity factor $f_{2}$ was higher than 36 with each formulation, indicating that the average difference between experimental and modeled plasma concentration profiles was less than $20 \%$. The largest deviation was observed at the peak plasma concentrations, where the model slightly underestimated the experimentally determined plasma concentrations. The values of $k_{g e}$ and $F_{a}$ obtained by regressing Eqs. 5-11 to the plasma concentration profiles are detailed in Table $\mathrm{V}$.

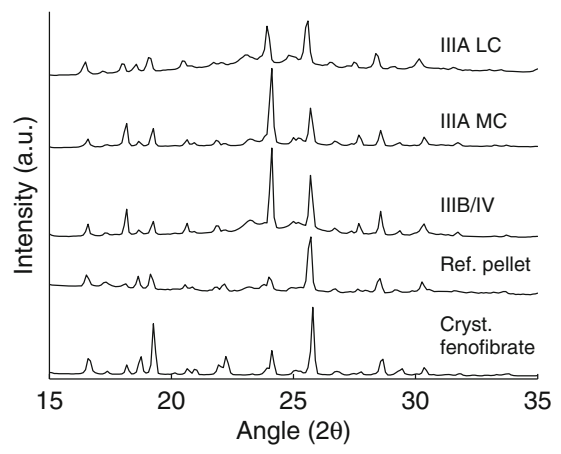

Fig. 6 XRPD pattern of the pellet phase obtained upon lipolysis of LBF IIIA LC, IIIA MC, and IIIB/IV. The reference was the pellet phase obtained from lipolysis of drug-free formulation, which was spiked with crystalline fenofibrate. The XRPD pattern of reference pellets were almost equal for formulation IIIA LC, IIIA MC, and IIIB/IV, and hence, only the pattern obtained from formulation IIIA MC is depicted. 

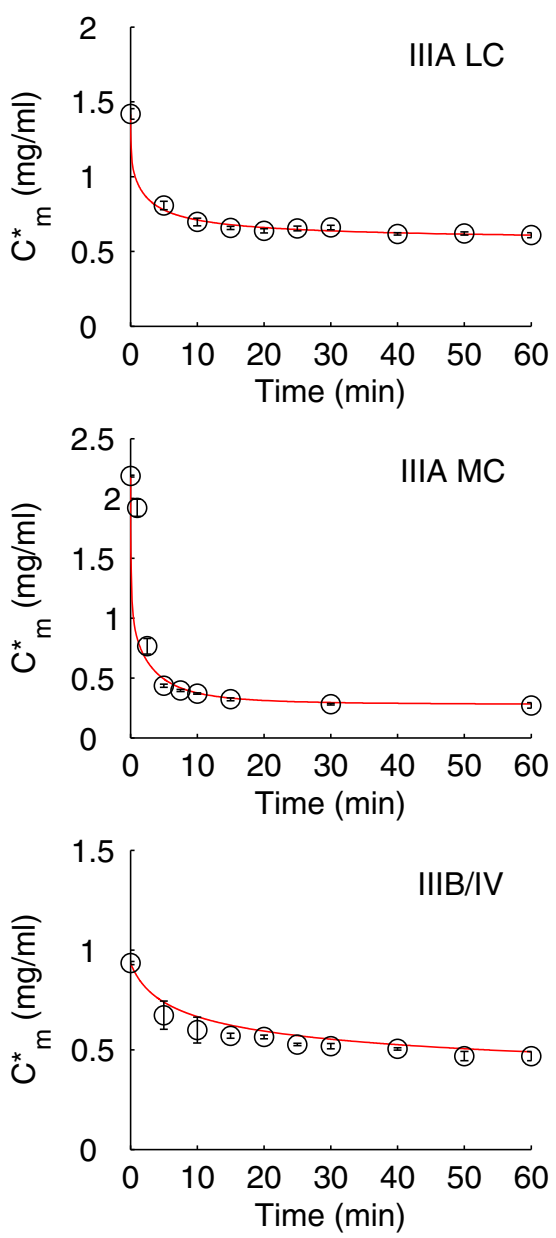

Fig. 7 Fenofibrate solubility during in vitro lipolysis of formulation IIIA LC, IIIA MC, and IIIB/NV. There was a very good agreement between experimental solubility data (circles) and the mathematical model of drug solubility (red lines), resulting in a $R^{2}$ of $0.9952,0.9941$, and 0.8930 for IIIA LC, IIIA MC, and IIIB/NV, respectively.

\section{Simulating Formulation Digestion and Drug Solubility in the Intestinal Lumen}

Using this model, the fenofibrate concentration profile in the intestinal lumen after p.o. administration of the three LBFs was estimated. Figure 9 a shows that maximum drug concentrations were reached $\sim 1 \mathrm{~h}$ after administration and that the drug was almost completely absorbed within $6 \mathrm{~h}$.

To simulate the amount of excipients in the intestinal lumen, the PK parameters obtained from in vivo data and the digestion

Table IV Relative fenofibrate solubility in dispersed TGs, SFs, LPI, and LP2 $\left(\mathrm{mg} \cdot \mathrm{ml}^{-1} \cdot \mathrm{mmol}^{-1}\right) \cdot \hat{C}_{L P 1}^{*}$ was estimated via parameter fitting (value $\left.\pm 95 \% \mathrm{Cl}\right)$

\begin{tabular}{lllll}
\hline Formulation & $\hat{C}_{T G}^{*}$ & $\hat{C}_{S F}^{*}$ & $\hat{C}_{L P 1}^{*}$ & $\hat{C}_{L P 2}^{*}$ \\
\hline IIIA LC & 0.631 & 0.640 & $0.369 \pm 0.001$ & 0.069 \\
IIIA MC & 0.701 & 0.640 & $0.093 \pm 0.011$ & 0.069 \\
IIIB/IV & $\mathrm{n} / \mathrm{a}$ & 0.640 & $\mathrm{n} / \mathrm{a}$ & 0.069 \\
\hline
\end{tabular}

rate of formulation lipids (calculated from in vitro lipolysis experiments) were utilized. The resulting time evolution of formulation lipids is depicted in Fig. 9 c-f. Low amounts of undigested TGs were available in the intestine, indicating that TGs were rapidly digested (Fig. 9c). In contrast, the amount of intralumenal SFs was notably higher, particularly for the Type IIIB/IV formulation (Fig. 9d). Figs. 9 e and f show the time course of LP1 and LP2 assuming no LPs absorption (continuous lines) and rapid LPs absorption $\left(P_{\text {eff,LP }}\right.$ of $10^{-4} \mathrm{~cm} / \mathrm{s}$, dotted lines). Because LPs absorption was much faster than formulation digestion, the LPs concentrations in the absorption environment were very low.

The time evolution of drug solubility in the intestinal lumen was then calculated based on the profiles of TGs, SFs, LP1, and LP2, and the relative fenofibrate solubilities listed in Table IV. Figure 9b displays the solubility profiles of the three LBFs assuming no LPs absorption (continuous lines) and rapid LPs absorption (dotted lines). During the first hour of intestinal transit, the drug solubility was similar for the three formulations (assuming no LPs absorption). At later time points, the solubility with formulation IIIB/IV was higher, followed by IIIA LC and IIIA MC. The impact of LPs absorption on drug solubility was particularly pronounced with formulation IIIA LC and IIIA MC, with substantially lower concentrations in the intestinal lumen under rapid LP absorptive conditions. In contrast for the Type IIIB/IV formulations the impact of LPs absorption was negligible.

\section{Drug Supersaturation in Intestinal Fluids}

The intralumenal drug concentration and solubility profiles were then used to calculate the $S R$ in the intestinal fluids (Eq. 36). This $S R$ profiles provided the basis for evaluating the risk of lipolysistriggered drug precipitation in an absorptive environment.

Figure 10a shows the profile of drug supersaturation assuming the nominal fenofibrate permeability $\left(P_{e f f, F F}\right.$ of $2.66 \cdot 10^{-5} \mathrm{~cm} / \mathrm{s}$ (13)) and rapid LPs absorption $\left(P_{\text {eff }, L P}\right.$ of $\left.10^{-4} \mathrm{~cm} / \mathrm{s}\right)$. We focused on the initial $6 \mathrm{~h}$ of intestinal transit as this was the estimated residence time of drug formulation in the gut (29). With the Type IIIB/IV and IIIA LC formulations, the highest SRs reached a value of $\sim 4$, but then rapidly decreased and fell below saturation after 1.5 and $3 \mathrm{~h}$, respectively. With formulation IIIA MC, the peak $S R$ value was slightly lower $(\sim 3)$, but the drug remained supersaturated for the longest period of time $(>4 \mathrm{~h})$.

In vitro lipolysis simulates conditions without an absorptive environment, and for comparison, it was interesting to calculate the time evolution of supersaturation in the intestinal lumen assuming no fenofibrate and LP absorption. As seen in Fig. 10b, each formulation became highly supersaturated. The rank-order of formulations obtained was similar to that observed with the in vitro lipolysis test, with highest $S R$ s for formulation IIIA MC, followed by IIIA LC, and IIIB/IV. The $S R$ s were particularly high with formulation IIIA MC where, after $6 \mathrm{~h}$ of digestion, values of $\sim 16$ were obtained. 
Fig. 8 Experimental (circles) and modeled (red lines) plasma concentration profiles obtained after administration of fenofibrate.
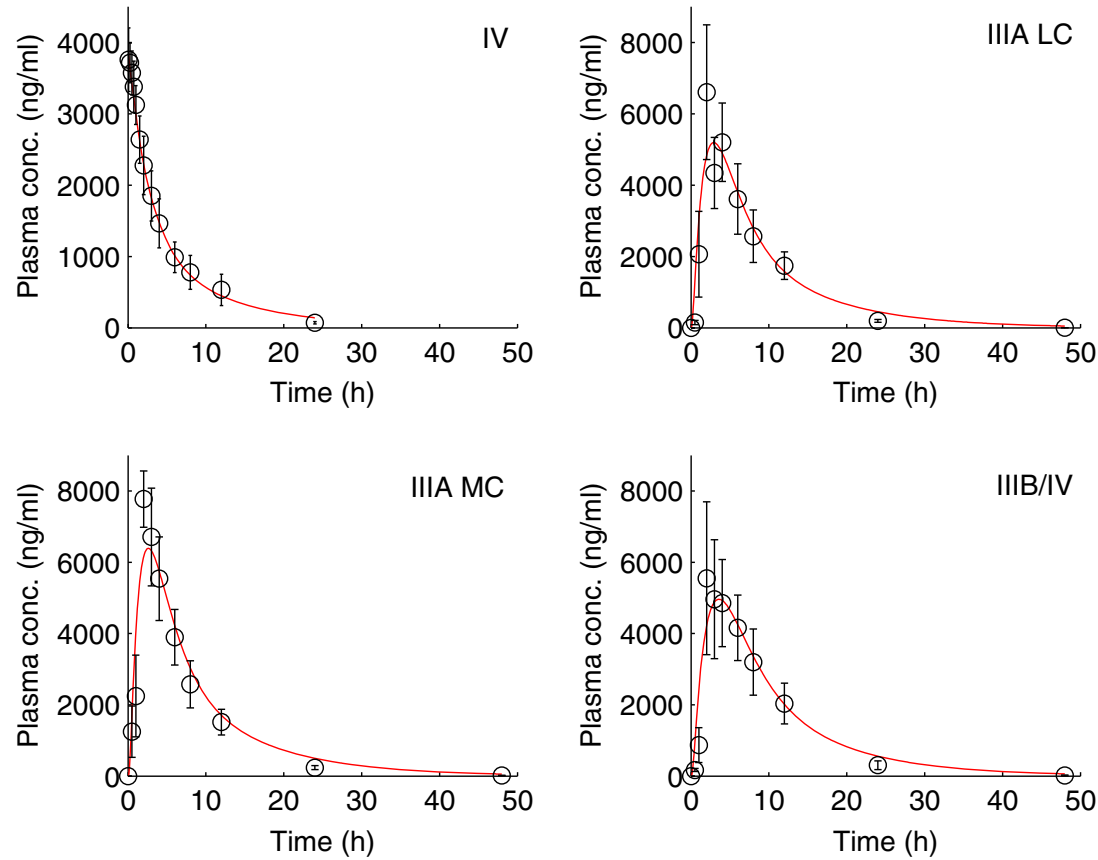

Impact of LPs Absorption on Drug Solubility and Supersaturation

In the previous simulation, rapid LPs absorption was assumed to simulate the worst case in terms of drug solubility. To better understand the influence of LPs absorption, we examined the intralumenal $S R$ assuming a range of different $P_{\text {eff, } L P}$ values. The result of this simulation is depicted in Fig. 11 for the absence of LPs absorption $\left(P_{\text {eff, } L P}=0 \mathrm{~cm} / \mathrm{s}\right)$ as well as for slow $\left(P_{\text {eff, } L P}=10^{-7} \mathrm{~cm} / \mathrm{s}\right)$, moderate $\left(P_{\text {eff, } L P}=10^{-5} \mathrm{~cm} / \mathrm{s}\right)$, and fast $\left(P_{\text {eff,LP }}=10^{-3} \mathrm{~cm} / \mathrm{s}\right)$ LPs absorption.

With formulations IIIA LC and IIIA MC, the intralumenal drug solubility was substantially lower when assuming high $P_{\text {eff,LP }}$ values $\left(>10^{-5} \mathrm{~cm} / \mathrm{s}\right)$. This effect was particularly pronounced with formulation IIIA LC (Fig. 11a), which was not only extensively digested, but also resulted in LPs with relatively high drug solubilization $\left(\hat{C}_{L P 1}^{*}: 0.369 \mathrm{mg} / \mathrm{ml}\right)$. In contrast, the solubilization capacity of medium-chain LPs was rather low $\left(\hat{C}_{L P 1}^{*}: 0.093 \mathrm{mg} / \mathrm{ml}\right)$ and, thus, the influence of LPs absorption on drug solubility was less pronounced (Fig. 11c). With formulation IIIB/IV, drug absorption was much faster than formulation digestion and, as a consequence, LPs absorption had a negligible influence on drug solubility and supersaturation (Fig. 11e).

The $S R$ in intestinal lumen as a function of time are shown in Fig. $11 \mathrm{~b}, \mathrm{~d}$, and $\mathrm{f}$. A notable increase in $S R$ was observed when $P_{\text {eff,LP }}$ exceeded $P_{\text {eff,FF, }}$, as seen with formulation IIIA LC and IIIA MC (red profiles in Fig. $11 \mathrm{~b}$ and d). In contrast, when drug absorption was much faster than formulation lipolysis and/or LPs absorption, the $S R$ was minimally affected by LPs absorption (Fig. 11f).

\section{Impact of Drug Permeability and Lipolysis Rate on Drug Supersaturation}

The influence of drug permeability and lipolysis rate on the extent of intralumenal supersaturation was subsequently evaluated. The supersaturation profile was determined assuming drug permeabilities, $P_{\text {eff }}$ in the range of 0.1 to 10 times the nominal value for fenofibrate $\left(2.66 \cdot 10^{-5} \mathrm{~cm} / \mathrm{s}\right)$ and assuming digestion rates in the range of 0.01 to 100 times the experimental lipolysis rate. The peak saturation ratios occurring during intestinal transit were then mapped as a function of $P_{\text {eff }}$ and the relative lipolysis rate (Fig. 12).

The resulting surface plots for the formulations IIIA LC and IIIA MC were significantly different from that of formulation IIIB/IV. With formulation IIIA LG and IIIA MC, the peak $S R$ s were highly dependent on the drug absorption and formulation lipolysis rates. The SRs became critically high (i.e., exceeding a value of $\sim 3$ (27) under low permeability conditions (i.e., $P_{\text {eff }}<10^{-5}$ ) and/or for relative lipolysis rates

Table V Pharmacokinetic parameters (value $\pm 95 \% \mathrm{Cl}$ ) estimated from plasma data after p.o. administration of the three LBFs (96 mg fenofibrate)

\begin{tabular}{lllll}
\hline LBF & $k_{\text {ge }}\left(h^{-1}\right)$ & $F_{a}(\%)$ & $f_{2}$ & $R^{2}$ \\
\hline IIIA LC & $0.555 \pm 0.327$ & $68.3 \pm 16.7$ & 37.9 & 0.8777 \\
IIIA MC & $0.681 \pm 0.347$ & $78.1 \pm 15.3$ & 41.8 & 0.9119 \\
IIIB/V & $0.377 \pm 0.178$ & $75.7 \pm 17.1$ & 42.9 & 0.8995 \\
\hline
\end{tabular}


Fig. 9 Time evolution of $\mathbf{a}$ fenofibrate concentration and $\mathbf{b}$ fenofibrate solubility, and of the amounts of $\mathbf{c} T \mathrm{TS}$, $\mathbf{d} \mathrm{SFs}$, e LPI, and $\mathbf{f} L P 2$ in the intestinal lumen upon p.o. administration of formulation IIIA LC (blue), IIIA MC (green), and IIIB/IV (red). Simulations were performed assuming no LPs absorption (continuous lines) and rapid LPs absorption (dotted lines). Only the first $6 \mathrm{~h}$ of $\mathrm{Gl}$ transit are depicted in the plots, which corresponds to the estimated gastric and small intestinal emptying time.
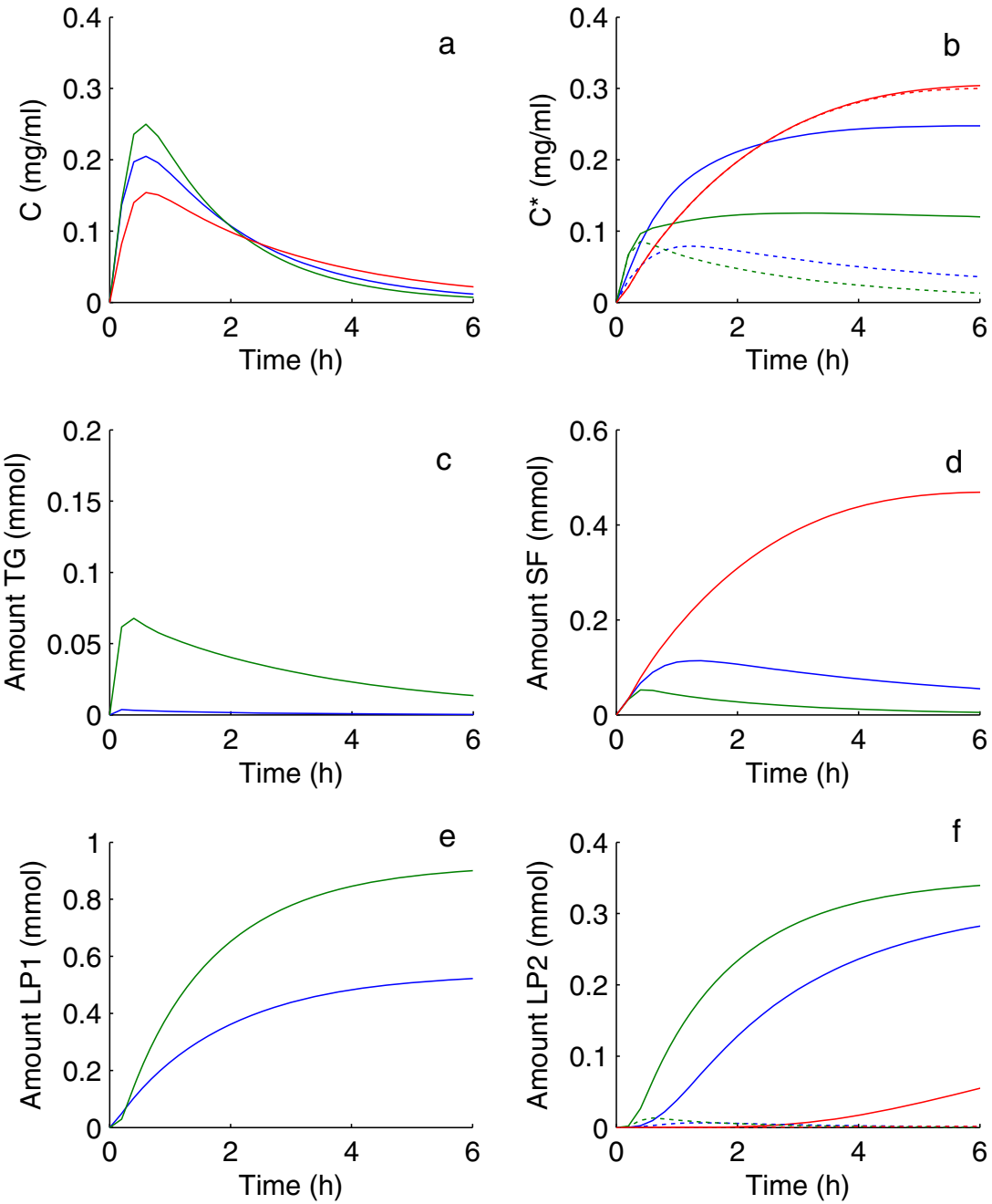

$>1$. In contrast, for high permeability and/or slow formulation lipolysis, the intestinal SRs remained at a constant low level of less than 4. With the IIIB/IV formulation, the impact of drug absorption and lipolysis rate on the intralumenal $S R$ was much less pronounced.

\section{DISCUSSION}

In the present study, we analyzed three fenofibrate-loaded LBFs that showed a poor correlation between formulation performance in vitro and in vivo. Using in vitro lipolysis testing without an absorption sink, all formulations resulted in extensive drug precipitation of crystalline material. This result suggested a notable loss in absorbable drug dose in the intestinal lumen. However, the PK profile obtained after p.o. administration of the LBFs to pigs showed that $\sim 70 \%$ of the administered drug dose was absorbed and no substantial difference in oral bioavailability was observed among the three formulations (15). Thus, it would appear that the extensive precipitation observed in vitro did not adversely impact drug absorption in vivo. Such poor correlations have been previously reported (10) and there is a clear need for more predictive in vitro assays for LBFs.

The aim of this study was to examine LBF lipolysis in an absorptive environment by introducing a predictive biopharmaceutical model of formulation digestion and drug absorption. This model revealed the drug supersaturation profile in the intestinal lumen, which provided a basis for estimating the risk of drug precipitation in vivo. It should be noted that this simulation was based on conservative assumptions, which resulted in worst-case prediction of in vivo LBF performance.

\section{Biopharmaceutical Model of Drug Supersaturation During LBF Digestion}

The novel biopharmaceutical model enabled analysis of drug supersaturation, and hence, of the risk of drug precipitation in the intestinal environment. A key aspect of supersaturation modeling was the simulation of drug solubility during intestinal transit of LBFs. To this end, we used a mathematical 
Fig. 10 Time evolution of the $S R$ in intestinal fluids assuming $\mathbf{a}$ sink conditions $\left(P_{\text {eff.FF }} 2.66 \cdot 10^{-5} \mathrm{~cm} / \mathrm{s}\right.$ and $\left.P_{\text {eff,LP }} 10^{-4} \mathrm{~cm} / \mathrm{s}\right)$ and $\mathbf{b}$ non-sink conditions for formulation IIIA LC (blue), IIIA MC (green), and IIIB/V (red). Only the first $6 \mathrm{~h}$ of $\mathrm{Gl}$ transit are depicted in the plots and the horizontal dotted lines correspond to a SR of I. a

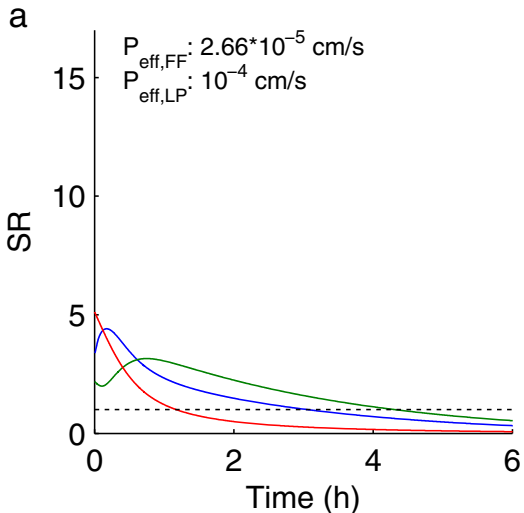

b

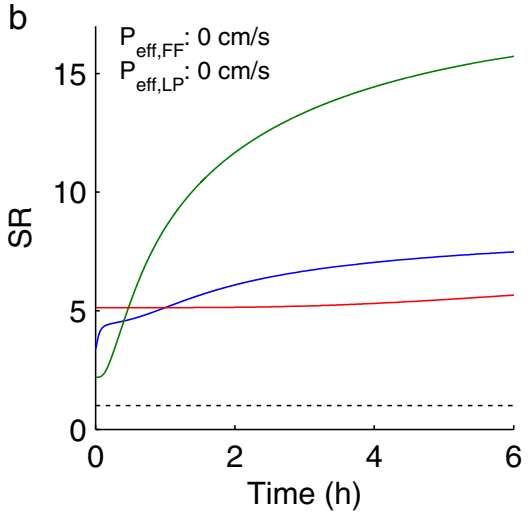

approach that was previously developed for modeling drug solubility in closed systems (14). However, drug solubilization in the GI tract is more dynamic and some adaptations were therefore necessary. The basic assumption was that each lipidic species (i.e., TGs, SFs, and LPs) contributed additively to drug solubilization at equilibrium and that there was a linear relationship between drug solubility and the concentration of excipients $(23,24)$. The resulting solubility model was validated
Fig. I I Influence of LPs absorption on intralumenal drug solubility (C*) and SR for formulations IIIA LC, IIIA MC, and IIIB/IV. Different $P_{\text {eff.LP }}$ values were assumed, which resulted in different LPs absorption rates, i.e., 0 (black), $10^{-7}$ (green), $10^{-5}$ (orange), and $10^{-3}$ (red) $\mathrm{cm} / \mathrm{s}$. The grey line shows the time evolution of intralumenal fenofibrate concentration assuming a $P_{\text {eff.FF }}$ of $2.66 \cdot 10^{-5} \mathrm{~cm} / \mathrm{s}$. Only the first 6 h of Gl transit are depicted in the plots and the horizontal dotted lines correspond to a SR of I.
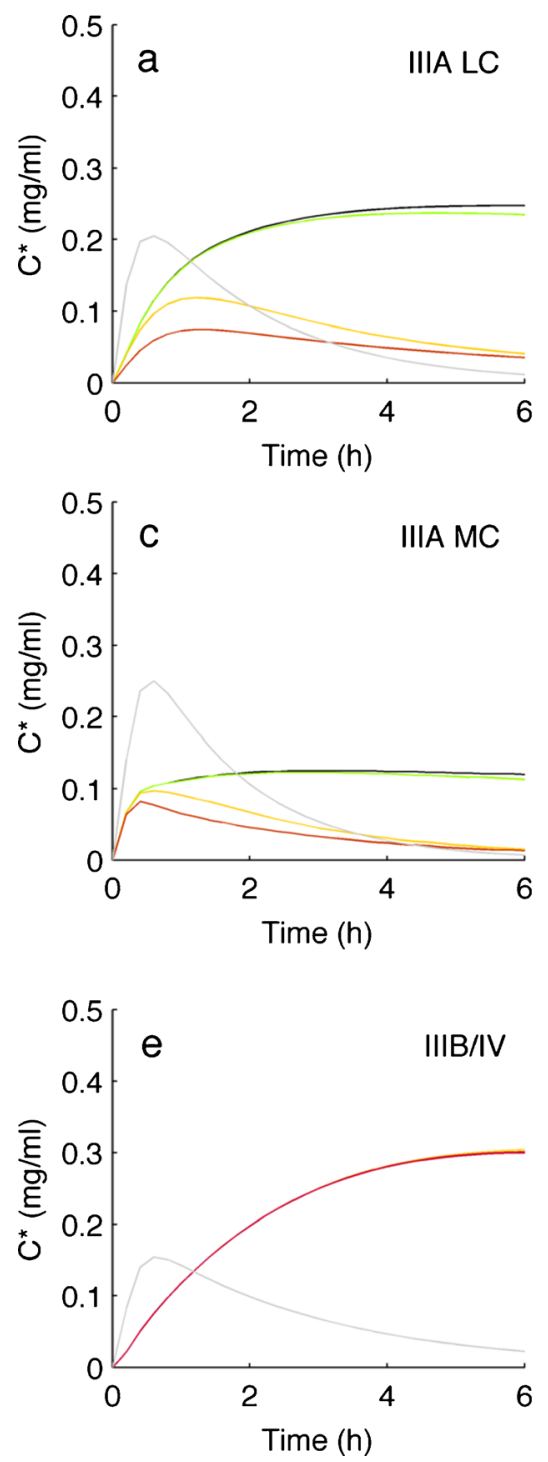
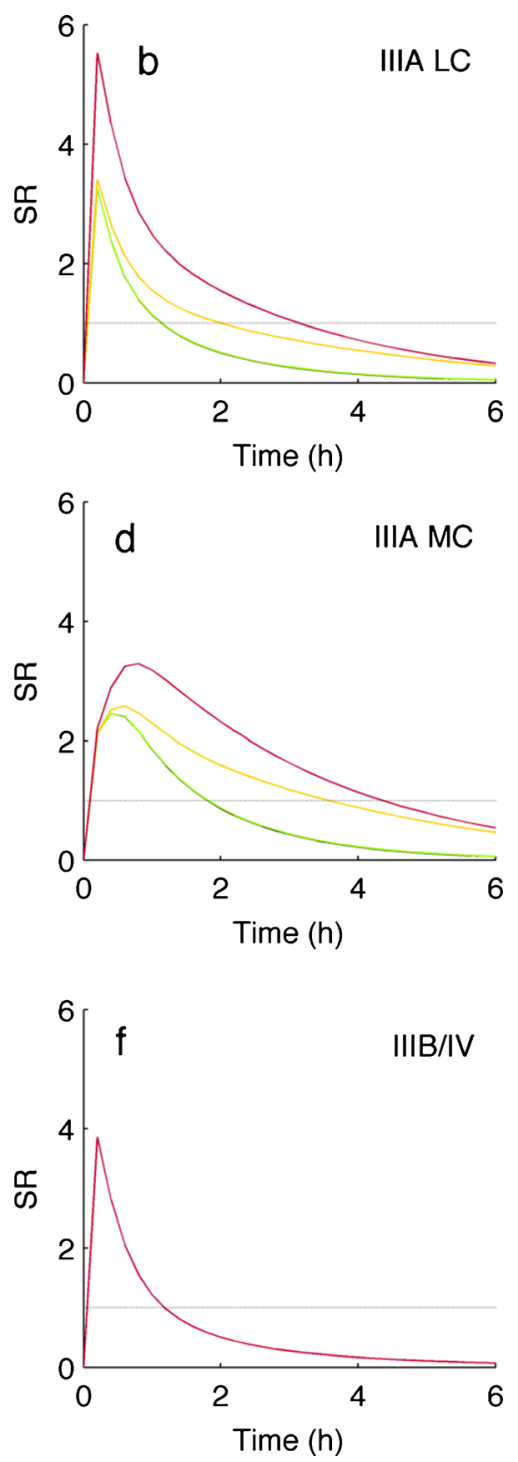
using experimental data obtained during in vitro lipolysis and resulted in an excellent correlation.

This strong correlation was a key requirement to simulate, in a second step, the time evolution of drug supersaturation in vivo. As a first evaluation, we simulated intestinal lipolysis with simultaneous drug and LPs absorption assuming the nominal fenofibrate permeability $\left(P_{\text {eff, } F F}: 2.66 \cdot 10^{-5} \mathrm{~cm} / \mathrm{s}\right)$ and rapid LPs absorption $\left(P_{e f f, L P}: 10^{-4} \mathrm{~cm} / \mathrm{s}\right)$. This $P_{\text {eff }, L P}$ value was deliberately selected to simulate worst-case in terms of drug solubilization capacity in the intestinal lumen. For comparison, we calculated the intralumenal supersaturation profile assuming no absorptive environment, which corresponded with the conditions used during routine in vitro lipolysis.

This comparison showed that the extent of fenofibrate supersaturation was dramatically lower assuming sink conditions than for non-sink conditions (Fig. 10a and b). For example, with formulation IIIA MC the peak $S R$ in a non-sink environment was around 15 , which indicated a highly unstable system and a substantial risk of drug precipitation. In contrast, assuming an absorption sink, the SRs were below 3. Similarly, the other formulations resulted in peak SRs in the range of 2.5 to 6 .

These smaller degrees of supersaturation certainly involved a much lower risk of drug precipitation compared to non-sink conditions. However, since the concentration of solubilized drug still exceeded the saturation level, the drug remained at risk for precipitation. It is important to consider that the model assumptions were simulating a worst-case situation. Some physiological parameters were associated with a relatively high degree of uncertainty and for these parameters conservative assumptions were employed (e.g., the surface expansion factor, $f$, and the volume of intestinal fluids, $V_{i}$ ). The volume of intestinal fluid in which the drug dose was diluted prior to absorption is particularly relevant in this regard. To our knowledge, no reference volume of intestinal fluids is available for pigs, and hence, we assumed a volume of $50 \mathrm{ml}$. This was the amount of water administered to the animals on formulation dosing and did not include intestinal secretions and the continuous access to water. A sensitivity analysis is straightforward in this case and demonstrates that the $S R$ is highly dependent from $V_{i}$. If for example the $V_{i}$ is doubled (i.e., $100 \mathrm{ml}$ ), which may reflect a more physiologically relevant scenario, the extent of supersaturation is divided in half resulting in SRs in the range of 1.25 to 3. Such values certainly suggest a substantially lower risk of drug precipitation.

A second aspect to consider is the definition of critical supersaturation. From a thermodynamic perspective, any saturation ratio $>1$ will lead to drug precipitation if sufficient time is given. The induction period for precipitation can be in the range of months to years for very low degrees of supersaturation, but becomes very short (seconds to minutes) when a critical range of supersaturation is exceeded (30). The critical supersaturation ratio is therefore very important from a biopharmaceutical perspective.
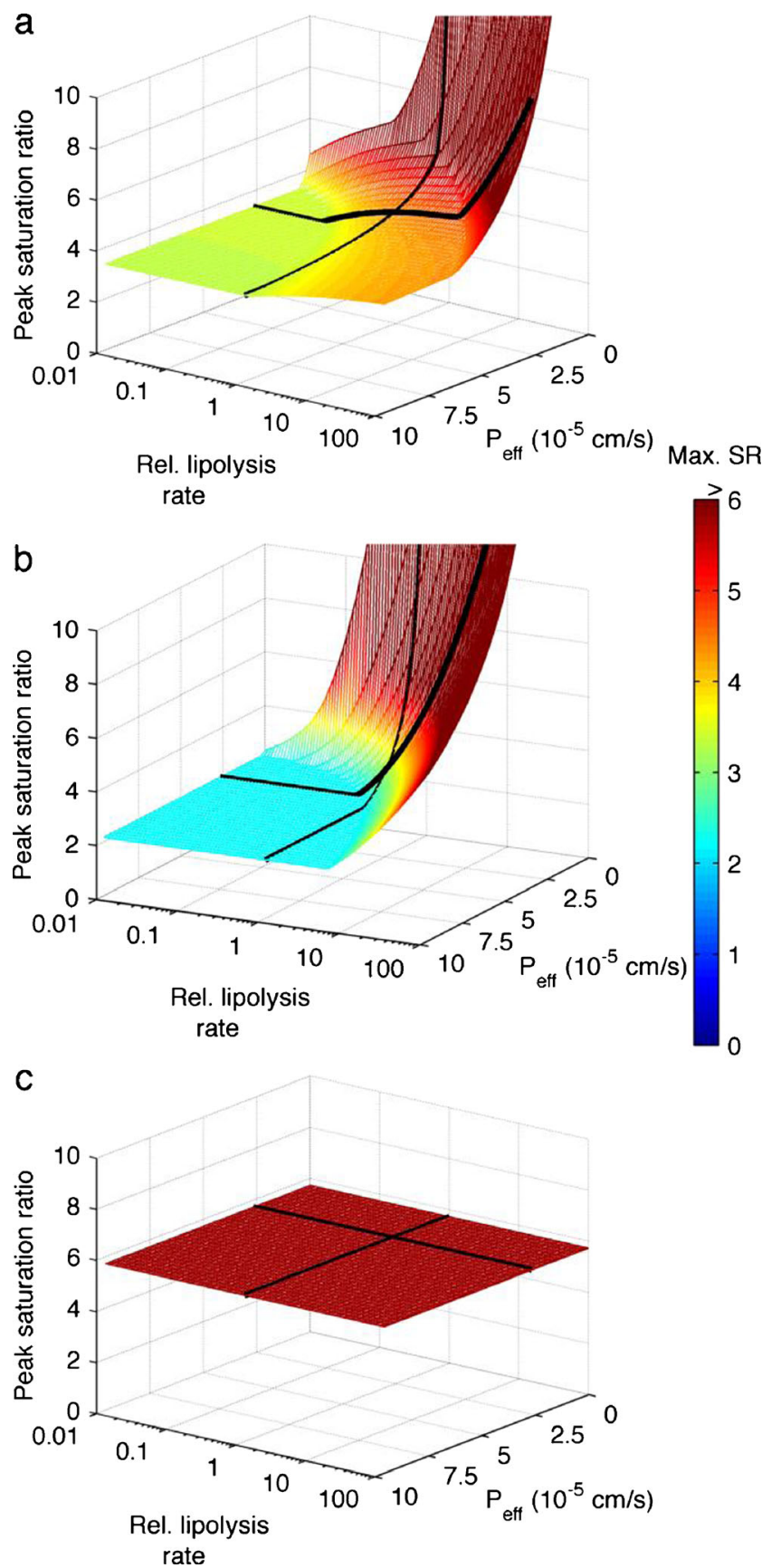

Fig. $123 \mathrm{D}$ surface plot showing the impact of drug permeability and lipolysis rate on the intralumenal peak saturation ratio for formulation a IIIA LC, b IIIA $\mathrm{MC}$, and $\mathbf{c} \| \mathrm{II} / \mathrm{V}$. The peak saturation ratios values corresponding to a $P_{\text {eff }}$ of $2.66 \cdot 10^{-5} \mathrm{~cm} / \mathrm{s}$ and to the experimental lipolysis rate are shown by black lines. The 3D surface plots were truncated for SR values higher than 10. With the extensively digested formulations IIIA LC (a) and IIIAMC (b), the resulting SRS were largely influenced by the rates of lipolysis and drug absorption. Assuming fast drug absorption (i.e., $P_{\text {eff }}>10^{-5}$ ), the peak $S R$ s remained below $\sim 4$ even for very fast digestion rates. In contrast, for slower absorption rates, the intralumenal supersaturation rapidly increased, particularly for relative lipolysis rates higher than one. With formulation IIIB/V the peak saturation ratio was not affected by the lipolysis and/or drug absorption rate (c).

Williams et al. recently addressed the importance of this parameter for drug precipitation during in vitro lipolysis (27). They proposed the $S R^{M}$ value as a measure for evaluating the 
risk of drug precipitation and found a critical threshold in the range of 2.5 to 3 . Consistent results were found for several drugs (danazol, fenofibrate, and tolfenamic acid) and different types of LBFs (LFCS type II-IV) (17, 27).

Care is certainly needed when applying a critical supersaturation ratio obtained from in vitro experiments to formulation lipolysis in vivo. The critical supersaturation ratio is, indeed, not the only determinant for drug precipitation in an absorptive environment. Important additional factors are the drug absorption rate and the duration of the induction period prior to precipitation. When the $S R$ exceeds a critical level, drug precipitation becomes highly probable, but the induction period still provides the opportunity for the drug to be absorbed. In case of a long induction period and/or fast drug absorption, the $S R$ can rapidly fall below the critical value and drug precipitation may ultimately be prevented. It is therefore likely that the driving force (i.e., the supersaturation ratio) must be higher for inducing drug precipitation in viwo than during in vitro lipolysis.

The biopharmaceutical model assumed that the drug was directly available for intestinal absorption once the formulation was dispersed. In this regard, we did not differentiate between drug that was solubilized in oil droplets, mixed micelles, and free drug in the aqueous bulk. Such a partitioning step is likely to have limited relevance on the overall drug absorption rate, as was recently suggested by Vertzoni et al. (31). However, there were other cases where only the free drug was directly available for absorption (32). This influence might be drug and formulation specific, and more studies are required to elucidate the individual mechanism of drug absorption. An additional drug partitioning step is expected to decelerate drug absorption. Neglecting this drug partitioning would therefore lead to an overestimation of the absorption rate. Such an overestimation was, however, not confirmed by the modeled data. On the contrary, the model described in vivo data very accurately with the exception of maximum plasma concentrations, which were slightly underestimated. However, due to this uncertainty, we also performed a parameter sensitivity analysis assuming a range of drug permeabilities, as will be discussed later in this section.

\section{Influence of LPs Absorption, Drug Permeability, and Lipolysis Rate on Intralumenal Drug Supersaturation}

The biopharmaceutical model of drug supersaturation provided the unique possibility to estimate the impact of LPs absorption on the solubilization capacity of LBFs during digestion, and hence, to assess the risk of lipolysis-induced precipitation under continuous absorptive conditions.

We analyzed the impact of LPs absorption as a parameter sensitivity analysis while assuming a range of different $P_{e f f, L P}$ values. Precise estimates of the effective permeability of LPs are difficult to obtain. One major reason is the fact that LPs are converted back into TGs in the enterocytes. Hence, conventional methods for permeability measurement cannot be applied in this particular case. Moreover, the flux of LPs across biological membranes might be influenced by the composition of the formulation (e.g., via the interaction with membrane transporters or the temporary residence of LPs at the oil-to-water interface).

We observed that the influence of LPs absorption on fenofibrate solubility varied substantially among the formulations. The most pronounced decrease in solubility was observed with formulation IIIA LC and IIIA MC. Formulation IIIA LC was rapidly digested and the long-chain LPs liberated from olive oil exhibited relatively high solubilization capacity. Hence, the removal of long-chain LPs had a pronounced influence on intestinal solubility. In contrast, the solubilization capacity of LPs liberated from Miglyol and from SFs was comparatively low and, although formulation IIIA MC was rapidly digested, there resulted a moderate influence of LPs removal on the overall drug solubility. The effect of LPs absorption on the $S R$ was largely determined by the relative rates of drug and LPS absorption. Indeed, the $S R$ was only affected when the absorption of LPs was faster than the absorption of fenofibrate. This observation indicated that, although the impact of LPs on the intestinal $S R$ was minimal for fenofibrate, it might be critical in case of poorly permeable drug compounds, especially when LPs provide substantial drug solubilization.

A comparatively different behavior was observed with formulation IIIB/IV. This formulation was digested slowly and the resulting LPs had low drug solubilization capacity. As a result, LPs absorption had almost no influence on intralumenal supersaturation.

It should also be noted that the relative solubilities $\hat{C}_{L P 1}^{*}$ and $\hat{C}_{L P 2}^{*}$ were obtained from drug solubility studies using digests from in vitro lipolysis. These values theoretically included the contribution of monoglycerides, monosorbitanesters, and FAs to drug solubilization. However, in the in vitro lipolysis model, the FAs are largely removed from solution via precipitation of calcium soaps and micellization with bile salts. The solubilization capacity of these precipitated FAs is expected to be lower than that of the solubilized products (3). Hence, these experimental solubilities may slightly underestimate the real solubilization capacity of digested formulations in vivo.

Finally, we examined the intralumenal $S R$ for different lipolysis and drug absorption rates (Fig. 12). This analysis was motivated by potential differences between the formulation lipolysis rates in vitro and in vivo. Similarly, the drug absorption rate could be influenced by the presence of lipidbased colloids, and thus, deviate from the nominal value of $2.66 \cdot 10^{-5} \mathrm{~cm} / \mathrm{s}$, as previously discussed. To examine how drug supersaturation is affected by these parameters, we mapped the peak saturation ratio occurring during intestinal transit as a function of both lipolysis rate and effective permeability. 
The results were significantly different among the three formulations. With formulation IIIB/IV the peak saturation ratio was not affected by the lipolysis and/or drug absorption rate (Fig. 12c). This result was due to the fact that formulation IIIB/IV became supersaturated upon simple dispersion (peak $S R=5.7$ assuming a $V_{i}$ of $50 \mathrm{ml}$ ) and, since formulation lipolysis was always slower than drug absorption, this $S R$ was never exceeded. Such a behavior might be typical for surfactantonly systems, for which formulation dispersion is generally more critical than formulation digestion (33).

In contrast, with the extensively digested formulations IIIA LC and IIIA MC, the resulting SRs were largely influenced by the rates of lipolysis and drug absorption (Figs. 12a and b, respectively). Assuming fast drug absorption (i.e., $P_{\text {eff }}>10^{-5}$ ), the peak SRs remained below $\sim 4$ even for very fast digestion rates. In contrast, for slower absorption rates, the intralumenal supersaturation rapidly increased, particularly for relative lipolysis rates higher than one.

These results suggested that, for highly permeable drugs, intestinal lipolysis is less critical regarding drug precipitation, even if the formulation is extensively digested. This situation is clearly different for drugs with low permeability characteristics. These compounds are at higher risk for lipolysis-triggered supersaturation and may result in relevant drug precipitation prior to absorption. With surfactant-only formulations, lipolysis generally plays a minor role, but drug precipitation can be induced by simple formulation dispersion, especially at higher drug loads. For this type of LBF, the in vitro-in silico-in vivo approach presented by Fei et al. might be appropriate to simulate formulation behavior in vivo, although it did not include formulation digestion (13).

\section{CONCLUSIONS}

This study introduced the first biopharmaceutical model of lipolysis-triggered drug supersaturation during intestinal LBF digestion. We analyzed three fenofibrate-loaded LBFs that resulted in extensive drug precipitation during in vitro lipolysis, but led to good oral bioavailability in vivo. The biopharmaceutical model showed that, for these formulations, the extent of supersaturation was considerably lower when lipolysis occurred in a continuous absorptive environment. This novel approach provided strong evidence of the importance of an absorption sink to estimate drug supersaturation, and hence, the risk of precipitation. Current in vitro assays simulate the worst-case with respect to drug precipitation, due to the absence of an absorption sink. Drug supersaturation is considerably higher than in the intestinal lumen and, as a consequence, drug precipitation is more likely to be observed in vitro.

Biopharmaceutical modeling was useful for gaining a deeper understanding of LBF performance in the GI tract.
For highly permeable compounds, drug absorption is rather fast compared to formulation digestion and critical degrees of supersaturation may therefore be avoided. Thus, the digestion of LBFs containing highly permeable drug compounds is less critical than previously assumed. This realization is highly encouraging with respect to the use of LBFs for oral administration of poorly water-soluble, but highly permeable drugs.

In the absence of in vitro tools simulating an absorption sink, such biopharmaceutical modeling should be further considered in LBF research and development. This strategy offers several opportunities for improving the prediction of LBF performance in vivo. A drug precipitation step could be included in the simulation and, to this end, the complex interplay between formulation digestion, drug supersaturation, and precipitation must be further investigated. Future biopharmaceutical modeling may also include biological effects of drug and excipients on oral absorption, such as the influence on efflux transporters or on the lymphatic drug transport.

\section{REFERENCES}

1. Porter CJH, Trevaskis NL, Charman WN. Lipids and lipid-based formulations: optimizing the oral delivery of lipophilic drugs. Nat Rev Drug Discov. 2007;6:231-48.

2. Shah NH, Carvajal MT, Patel CI, Infeld MH, Malick AW. Selfemulsifying drug-delivery systems (SEDDS) with polyglycolyzed glycerides for improving in-vitro dissolution and oral absorption of lipophilic drugs. Int J Pharm. 1994;106:15-23.

3. Devraj R, Williams HD, Warren DB, Mullertz A, Porter CJH, Pouton CW. In vitro digestion testing of lipid-based delivery systems: Calcium ions combine with fatty acids liberated from triglyceride rich lipid solutions to form soaps and reduce the solubilization capacity of colloidal digestion products. Int J Pharm. 2013;441:323-33.

4. Sassene PJ, Knopp MM, Hesselkilde JZ, Koradia V, Larsen A, Rades $\mathrm{T}$, et al. Precipitation of a poorly soluble model drug during in vitro lipolysis: characterization and dissolution of the precipitate. J Pharm Sci. 2010;99:4982-91.

5. Thomas N, Holm R, Mullertz A, Rades T. In vitro and in vivo performance of novel supersaturated self-nanoemulsifying drug delivery systems (super-SNEDDS). J Control Release. 2012;160:25-32.

6. Brouwers J, Brewster ME, Augustijns P. Supersaturating drug delivery systems: the answer to solubility-limited oral bioavailability? J Pharm Sci. 2009;98:2549-72.

7. Yeap YY, Trevaskis NL, Porter CJH. The potential for drug supersaturation during intestinal processing of lipid-based formulations may be enhanced for basic drugs. Mol Pharmaceutics. 2013;10: 2601-15.

8. Sek L, Porter CJH, Charman WN. Characterisation and quantification of medium chain and long chain triglycerides and their in vitro digestion products, by HPTLC coupled with in situ densitometric analysis. J Pharmaceut Biomed. 200 1;25:651-61.

9. Zangenberg NH, Mullertz A, Kristensen HG, Hovgaard L. A dynamic in vitro lipolysis model I. Controlling the rate of lipolysis by continuous addition of calcium. Eur J Pharm Sci. 2001;14:115-22.

10. Fatouros DG, Mullertz A. In vitro lipid digestion models in design of drug delivery systems for enhancing oral bioavailability. Expert Opin Drug Metab Toxicol. 2008;4:65-76. 
11. Shi Y, Gao P, Gong YC, Ping HL. Application of a biphasic test for characterization of in vitro drug release of immediate release formulations of celecoxib and its relevance to in vivo absorption. Mol Pharmaceutics. 2010;7:1458-65.

12. Kataoka M, Sugano K, Mathews CD, Wong JW, Jones KL, Masaoka Y, et al. Application of dissolution/permeation system for evaluation of formulation effect on oral absorption of poorly watersoluble drugs in drug development. Pharm Res. 2012;29:1485-94.

13. Fei Y, Kostewicz ES, Sheu MT, Dressman JB. Analysis of the enhanced oral bioavailability of fenofibrate lipid formulations in fasted humans using an in vitro-in silico-in vivo approach. Eur J Pharm Biopharm. 2013;85:1274-84.

14. Stillhart G, Imanidis G, Kuentz M. Insights into drug precipitation kinetics during in vitro digestion of a lipid-based drug delivery system using in-line Raman spectroscopy and mathematical modeling. Pharm Res. 2013;30:3114-30.

15. Griffin BT, Kuentz M, Vertzoni M, Kostewicz ES, Fei Y, Faisal W, Stillhart C, O'Driscoll C, Reppas C, Dressman JB. Comparison of in vitro tests at various levels of complexity for the prediction of in vivo performance of lipid-based formulations. Eur J Pharm Biopharm. 2014;86(3):427-37.

16. Geladi P, Kowalski BR. Partial least-squares regression - A tutorial. Anal Chim Acta. 1086;185:1-17

17. Anby MU, Williams HD, McIntosh M, Benameur H, Edwards GA, Pouton CW, et al. Lipid digestion as a trigger for supersaturation: evaluation of the impact of supersaturation stabilization on the in vitro and in vivo performance of self-emulsifying drug delivery systems. Mol Pharmaceutics. 2012;9:2063-79.

18. NajibJ. Fenofibrate in the treatment of dyslipidemia: A review of the data as they relate to the new suprabioavailable tablet formulation. Clin Ther. 2002;24:2022-50.

19. Kararli TT. Comparison of the gastrointestinal anatomy, physiology, and biochemistry of humans and commonly used laboratory animals. Biopharm Drug Dispos. 1995;16:351-80.

20. Avdeef A, Tam KY. How well can the Caco-2/Madin-Darby canine kidney models predict effective human jejunal permeability? J Med Chem. 2010;53:3566-84.

21. Jantratid E, Janssen N, Reppas C, Dressman JB. Dissolution media simulating conditions in the proximal human gastrointestinal tract: An update. Pharm Res. 2008;25:1663-76.

22. Shah VP, Tsong Y, Sathe P, Liu JP. In vitro dissolution profile comparison - Statistics and analysis of the similarity factor, $\mathrm{f}_{2}$. Pharm Res. 1998;15:889-96.
23. Yalkowsky SH. Solubility and solubilization in aqueous media. Washington NY: Oxford University Press; 1999. pp. 236-320.

24. Grove M, Pedersen GP, Nielsen JL, Mullertz A. Bioavailability of seocalcitol I: Relating solubility in biorelevant media with oral bioavailability in rats - Effect of medium and long chain triglycerides. J Pharm Sci. 2005;94:1830-8.

25. Fernandez S, Jannin V, Rodier JD, Ritter N, Mahler B, Carrière F. Comparative study on digestive lipase activities on the self emulsifying excipient Labrasol ${ }^{\circledR}$, medium chain glycerides and PEG esters. Biochim Biophys Acta Mol Cell Biol Lipids. 2007;1771:633-40.

26. Fernandez S, Rodier JD, Ritter N, Mahler B, Demarne F, Carrière $\mathrm{F}$, et al. Lipolysis of the semi-solid self-emulsifying excipient Gelucire ${ }^{\circledR}$ 44/14 by digestive lipases. Biochim Biophys Acta Mol Cell Biol Lipids. 2008;1781:367-75.

27. Williams H, Sassene P, Kleberg K, Calderone M, Igonin A, Jule E, et al. Toward the establishment of standardized in vitro tests for lipidbased formulations, Part 3: Understanding supersaturation versus precipitation potential during the in vitro digestion of type I, II, IIIA, IIIB and IV lipid-based formulations. Pharm Res. 2013;30: 3059-76.

28. Williams HD, Sassene P, Kleberg K, Bakala-N'Goma JC, Calderone $\mathrm{M}$, Jannin $\mathrm{V}$, et al. Toward the establishment of standardized in vitro tests for lipid-based formulations, part 1: Method parameterization and comparison of in vitro digestion profiles across a range of representative formulations. J Pharm Sci. 2012;101:3360-80.

29. Sugano K. Biopharmaceutics modeling and simulations. Hoboken NJ: Wiley; 2012.

30. Mullin JW. Crystallization. Oxford: Butterworth-Heinemann; 2001.

31. Vertzoni M, Markopoulos G, Symillides M, Goumas C, Imanidis G, Reppas C. Luminal lipid phases after administration of a triglyceride solution of danazol in the fed state and their contribution to the flux of danazol across Caco-2 cell monolayers. Mol Pharmaceutics. 2012;9:1189-98.

32. Miller JM, Beig A, Krieg BJ, Carr RA, Borchardt TB, Amidon $\mathrm{GE}$, et al. The solubility-permeability interplay: mechanistic modeling and predictive application of the impact of micellar solubilization on intestinal permeation. Mol Pharmaceutics. 2011;8:1848-56.

33. Mohsin K, Long MA, Pouton CW. Design of lipid-based formulations for oral administration of poorly water-soluble drugs: precipitation of drug after dispersion of formulations in aqueous solution. J Pharm Sci. 2009;98:3582-95. 